\title{
Blockchain and artificial intelligence technology in e-Health
}

\author{
Priti Tagde $^{1,2} \cdot$ Sandeep Tagde ${ }^{2}$. Tanima Bhattacharya ${ }^{3,4} \cdot$ Pooja Tagde $^{5} \cdot$ Hitesh Chopra $^{6} \cdot$ Rokeya Akter $^{7}$. \\ Deepak Kaushik $^{8} \cdot$ Md. Habibur Rahman ${ }^{9}$ (D)
}

Received: 27 May 2021 / Accepted: 24 August 2021 / Published online: 2 September 2021

(C) The Author(s), under exclusive licence to Springer-Verlag GmbH Germany, part of Springer Nature 2021

\begin{abstract}
Blockchain and artificial intelligence technologies are novel innovations in healthcare sector. Data on healthcare indices are collected from data published on Web of Sciences and other Google survey from various governing bodies. In this review, we focused on various aspects of blockchain and artificial intelligence and also discussed about integrating both technologies for making a significant difference in healthcare by promoting the implementation of a generalizable analytical technology that can be integrated into a more comprehensive risk management approach. This article has shown the various possibilities of creating reliable artificial intelligence models in e-Health using blockchain, which is an open network for the sharing and authorization of information. Healthcare professionals will have access to the blockchain to display the medical records of the patient, and AI uses a variety of proposed algorithms and decision-making capability, as well as large quantities of data. Thus, by integrating the latest advances of these technologies, the medical system will have improved service efficiency, reduced costs, and democratized healthcare. Blockchain enables the storage of cryptographic records, which AI needs.
\end{abstract}

Keywords Blockchain $\cdot$ Artificial intelligence $\cdot$ Electronic health records $\cdot e-H e a l t h \cdot$ Data security

\section{Introduction}

The health informatics technique underlying budgeting, personnel, patients, legal disputes, logistics, supplies, and other procedures and medical workflows are often made up of a sequence of conditional steps that can be visualized as a series of repeated patient-care activities (Alotaibi and Federico 2017). Among hospitals and other healthcare service providers, internal controls should be increased; performance, compliance, and consistency should be enhanced; and risk,

\section{Responsible Editor: Lotfi Aleya}

Priti Tagde

tagde_priti@rediffmail.com

Md. Habibur Rahman

pharmacisthabib@gmail.com

1 Bhabha Pharmacy Research Institute, Bhabha University Bhopal, Bhopal M.P, India

2 PRISAL Foundation (Pharmaceutical Royal International Society), New delhi, India

3 School of Chemistry \& Chemical Engineering, Hubei University, Wuhan, China job time, and overhead should be reduced (Chapuis et al. 2010). This article outlines a healthcare smart contract structure that can handle patient data and simplify complicated medical treatments (Campanella et al. 2016), based on advanced healthcare blockchain analysis and a robust approach to healthcare management. We have looked at cutting-edge blockchain studies in healthcare and presented a blockchainbased solution to healthcare management. As shown by numerous initiatives in various countries and economies, governments and related business sectors are becoming more

4 Department of Science \& Engineering, Novel Global Community Education Foundation, Hebersham, Australia

5 Practice of Medicine Department, Govt. Homeopathy College, Bhopal, M.P, India

6 Chitkara College of Pharmacy, Rajpura, Punjab 140401, India

7 Department of Pharmacy, Jagannath University, Sadarghat, Dhaka 1100, Bangladesh

8 Department of Pharmaceutical Sciences, Maharshi Dayanand University, Rohtak, Haryana 124001, India

9 Department of Pharmacy, Southeast University, Banani, Dhaka 1213, Bangladesh 
involved in digitizing healthcare systems. The path to success is to integrate technology into each company's DNA by utilizing blockchain, AI, and other accessible technologies (Wong et al. 2019; Bragazzi et al. 2020). To advance medical research and achieve patient-centricity, the industry would use technology to create user- and customer-centric interfaces and data-driven decisions for innovative data processing approaches and better results (Sahoo and Baruah 2018). For example, artificial intelligence (AI) could help identify and prioritize individual patients for drug monitoring and growth, critical for managed drug production and shorter timelines (Paul et al. 2021). For repurposing marketed medications, researching the effectiveness of medication formulations, and dose measurement, clinical trial data was monitored using numerical drug design methods and $\mathrm{AI}$ (Cha et al. 2018). As a consequence of such a rapidly evolving climate, governments must identify the most effective ways to leverage resources and drive reform while ensuring the necessary consistency, compliance, or data protection (Siyal et al. 2019). We have mention in Fig. 1 security measures in e-Health by blockchain and AI. Blockchain aids in the creation of a system that develops and manages content blocks known as ledgers, with safe and automated data analysis. All health-related data will be safely recorded and analyzed, allowing medical experts, healthcare providers, and payers to receive timely updates. This is taken a step further by AI algorithms into the blockchain (Hang et al. 2019).To comprehend health trends and patterns, AI began to think and learn like a clinician. It gathers information from various sources, including the patient, the radiologist, and images in the form of unstructured data.

$\mathrm{AI}$ is also capable of executing complicated computational functions and quickly evaluates vast volumes of patient details. However, some doctors remain cautious using AI in healthcare, particularly in positions that may influence a patient's wellbeing, considering the powerful capabilities that AI can provide, which has demonstrated that, quicker than a person, it can execute many dynamic and cognitive functions. The automobile industry has already proven its ability to exploit AI to deliver driverless vehicles. However, other companies have already discovered ways to identify fraud or determine financial threats by utilizing machine learning. Only a few points are demonstrating AI's maturity level (Akkiraju et al. 2020).

\section{Blockchain in e-Health}

The blockchain is a distributed database using state machine replication, with atomic changes to the database referred to as transactions grouped into blocks, with the integrity and tamper-resistance of the transaction log assured via hash links among blocks. The blockchain concept was introduced for Bitcoin in the context of decentralized electronic currency. The popularity of Bitcoin allows blockchain that can use without depending on any established third party to enable trustworthy and safe transactions through an untrusted network. There have been many reports on basic building blocks in the blockchain (Feng et al. 2019). A sequential series of blocks containing a list of complete and correct transaction records is blockchain. The blocks are connected by a relation (hash value) to the previous block, thereby creating a chain (Lin et al. 2020). The block that precedes a given block is recognized as its block header, and the very first block is recognized as the block of genesis (Ahmad et al. 2019; Zhang et al. 2020). With a growing interest in many applications, spanning from data storage, financial markets, computer protection, IoT, and nutritional science to the healthcare sector and brain studies, blockchain technology has gained tremendous popularity and progress to distribute safe and stable monitoring of healthcare records. It may be a tool in the future that could theoretically assist in customized, credible, and safe healthcare by combining and displaying the whole real-time clinical
Fig. 1 Block chain and artificial intelligence security in e-Health

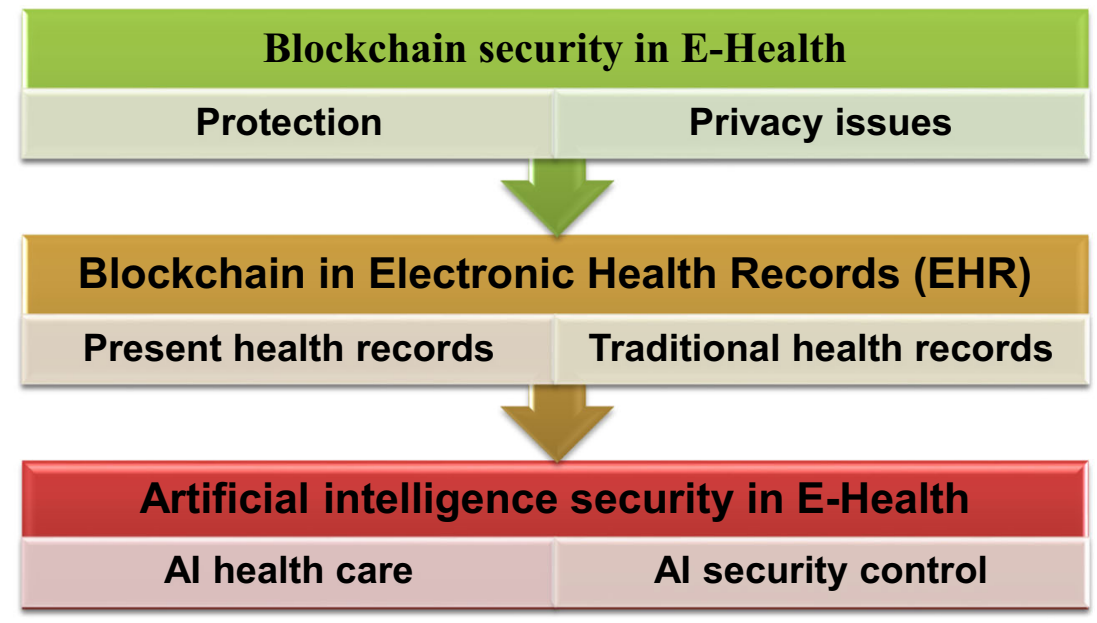


records of a patient's wellbeing in an up-to-date, secure healthcare setup (Linn and Koo 2016).

Blockchain platform to determine the health care status of patient illnesses, focused on concurrent execution and artificial intelligence healthcare networks and the proposed approach analyses of the patient's overall state, diagnosis, and recovery system, and investigates the relevant surgical interventions by simultaneous operations and clinical decisionmaking computational studies to assess the quality of care for patients and the feasibility of diagnosis, the proposed method has been evaluated in actual, as well as simulated, healthcare systems (Bryatov and Borodinov 2019; Hylock and Zeng 2019).

Blo CHIE, a specific blockchain-oriented healthcare knowledge-sharing network, was created by Singh and Kim (2018), and the proposed platform evaluates the criteria for the exchange of health care data, specifically for personal health data and electronic medical reports, and discusses numerous other types of data by integrating blockchains within various sources. They combined the application with on-chain and off-chain authentication processes to verify the good specifications of both validity and privacy. It is possible to significantly increase the implementation of blockchain technologies to exchange medical records, anonymity, and protection between clinical professionals and healthcare institutions (Andoni et al. 2019).

Cryan, M.A. adopting a related mechanism (Khurshid 2020) indicated a systematic and creative infrastructure leveraging blockchain technologies to secure confidential patient records, solve fundamental data protection problems, and introduce a hospital-wide blockchain software framework. In biomedical science and therapeutic domains, blockchain technology has also shown tremendous promise. Through the practical application of blockchain technologies, even before beginning a clinical study or test, it may be possible to store all clinical approvals, schedules, and protocols on a blockchain. In this way, important clinical trial-related details will become more up-to-date, protected, time-stamped, and openly transparent.

\section{Blockchain healthcare use cases in e-Health}

\section{Supply chain transparency}

The assurance of medical commodities' provenance to determine their legitimacy is a primary concern in the healthcare industry, as it is in many others. Customers may track items from the manufacturing site to each stage of the supply chain using a blockchain-based system, giving them complete visibility and transparency over what they are buying (Narayanaswami et al. 2019). This is a severe problem for the industry, especially in poor countries, where counterfeit prescription drugs cause tens of thousands of fatalities each year (Edwin et al. 2017). It is also becoming more critical for medical equipment; rapidly expanding as more remote health monitoring is implemented, attracting unscrupulous actors' interest.

MediLedger is a well-known blockchain system that allows businesses in the prescription drug supply chain to check the legality of medicines and expiration dates and other crucial information (Khezr et al. 2019).

\section{Smart contracts}

Smart contracts may also be deployed, repeated, and then enforced to maintain transparency across multiple stages of a medical study. A smart contract is a script that is processed on a blockchain in the sense of the blockchain (Xia et al. 2017). It was determined that a blockchain-based telemonitoring healthcare system for remote patients detects and manages cancerous cells. Smart contracts are included in the proposed procedure, along with blockchains that can be used widely to guarantee the authenticity and confidentiality of patient data in significantly advanced hospitals and outpatient residences.

Another report proposed Dermonet, a blockchain-based form of online dermatological consulting with a teledermatology surveillance device to assist patients with dermatology (Mannaro et al. 2017).

Proactive aging (Brandon et al. 1991), on the other side, is a blockchain-based network that helps promote aging people's active living. It can be remembered that blockchain technology may be an appropriate and well-suited solution in comprehensive medical care procedures, such as chronic conditions (e.g., cancer), surgical interventions, and aging. Moreover, to perform advanced transnational analysis at the genomic stage, it may be feasible for drug companies, drug producers, and biomedical researchers to utilize DNA knowledge housed on blockchains.

Smart contracts should introduce the blockchain to allow transfers among different people quicker and much more efficiently (Feingold and Fein 1987). In 1994, Nick Szabo introduced this idea. He described a smart contract as "allow computers to communicate procedure that implements the conditions of a contract" and proposed that contract provisions be converted to the code, thus reducing the need for counterparties to work between the groups (Rallapalli 2016).

In a blockchain, smart contracts have a destination identifier (Cong and He 2019). For trade, a smart contract may be rendered quickly by specifying the blockchain address. It is performed on each network node separately and manually, as determined by the data found in the transaction (Dubovitskaya et al. 2017a).

\section{Patient-centric electronic health records}

Over the past few years, medical professionals, clinics, and healthcare devices have been responsible for the need for a sheer rise in digital technology of medical health data, as 
digital technology of this knowledge facilitates more accessible communication and control and is also a foundation for better and faster decision-making. Currently, the most popular implementations of healthcare blockchain technology are in electronic medical records (Feingold and Fein 1987). Even so, electronic health records (EHRs) are never generated between various organizations to manage lifelong records, and individuals leave their data fragmented between several institutions when life circumstances isolate them from the data of one provider through another; therefore, they lose simple access to past data (Rallapalli 2016).

Many academics have brought up blockchain application verification to preserve the EHRs, having confronted a crucial need for a creative way to treat EHRs in a way that allows patients to share in their present and traditional health records. In handling security, honesty, and fast data exchange, a prototype called "MedRec" uses distinctive blockchain advantages (Wang et al. 2018). It operates on a decentralized basis for maintaining data and claims and provides patients with a complete, permanent background, providing quick and easy access to their particular clinical documents through multiple providers and care institutions (Scekic et al. 2019). "MedRec" would not preserve or trigger adaptation duration for medical documents. It stores a record label on a blockchain and informs the patient who is liable for where the form will go.

Medical data exchange also poses crucial drawbacks during the implementation of EHR, such as lack of influence over data, data provenance, monitoring, and safety monitoring of medical information during the deployment of EHR. It was launched MeD Share as a secured blockchain framework for sharing medical data between untrusted parties, keeping in mind certain limitations. MeD Share may be used to share medical data and retain electronic health records with high information authenticity, customized audit authority, and limited potential risks to data protection and privacy across cloud service providers, physicians, and healthcare research agencies. In general, EHR includes extremely sensitive and critical patient-related data, which is also exchanged for successful evaluation and treatment by physicians, neurosurgeons, healthcare professionals, physicians, and scientists (Cyran 2018).

When a hospital visit begins, most clinicians with that patient appear to see things immediately. Medication glitches, hypersensitivities, and medication solutions may be accommodated reasonably easily overall blockchain records by practical patient-caring algorithms without the need for cumbersome types of pharmaceutical compromise. Consequently, the usage of blockchain innovation would promote better access to treatment, monitoring of medical records (Mandl 2001), and timely confirmation of clinical knowledge, enhanced surveillance, and more productive organization of care.

\section{Medical staff credential verification}

Cryptographic protocols can track the experience of medical professionals in the same way that can use it to track the provenance of a medical good. Trusted medical institutions and healthcare organizations can log the credentials of their staff, which helps to streamline the hiring process for healthcare organizations. ProCredEx, based in the USA, has created a medical credential verification system based on the R3 Corda blockchain protocol (Funk et al. 2018).

The following are the main advantages of the blockchain system (Funk et al. 2018):

(a) During the employment process, healthcare organizations will be able to get credentialed more quickly.

(b) A chance for medical institutions, insurers, and healthcare providers to profit from their existing credentials data on previous and current employees.

(c) Transparency and assurance for partners, such as organizations that subcontract locum tenens or in emerging virtual health delivery models, tell patients about medical staff experience.

The patient's care may be affected during the collection, delivery, and dissemination of this highly confidential patient information to multiple organizations, which may pose significant risks to patient welfare and establish an up-to-date patient background. In chronically ill patients (e.g., leukemia and HIV), the incidence of such threats may increase due to a long history of pre-and post-treatment, obey, and recovery. Therefore, it has been particularly imperative to preserve an up-to-date medical background (Chou et al. 2015). Estonia debuted with worldwide supremacy in blockchain technology in 2016 when it introduced the concept of holding millions of medical documents confidential and having them publicly accessible to medical practitioners and insurance agencies at the same time and possibly the explanation for global development in the usage of blockchain technology in medicine is an explicit guarantee for patients to use this technology to make their own. All in the blockchain, any access or modification attempt can be easily labeled and remembered. This is useful for the integrity of patients and identifies any criminal activities, including wholesale fraud or record adulteration (Evans 1993).

\section{lot security for remote monitoring}

The use of remote monitoring solutions, in which all types of sensors detecting patients' vital signs are utilized to help give healthcare practitioners better visibility into patients' health, enabling more proactive and preventative care, is one of the largest developments in digital health (Khan and Byun 2020). 
However, security is a major concern in health IoT, both in keeping patient data private and secure and preventing it from being tampered with to generate misleading data. In some scenarios, where a connected device may be relied on in an emergency, such as alerting an elderly person's caregiver that they have fallen or had a heart attack, it is equally critical that the supporting systems are highly resilient to DDoS other attacks disrupting service.

Blockchain technology could aid in the secure monitoring of IoT devices in the field:

(a) Personal data is saved on the blockchain as a unique hash function, and blockchain cryptography ensures that only authorized parties have access to it (any change in the source data will create a different hash function, and a user must have a specific set of cryptographic keys to decode the hash function into the source data)

(b) It is practically hard to tamper with patient data after it is recorded on the blockchain ledger (as a hash function) because doing so would necessitate having access to all stored copies.

\section{Tracking clinical trials and pharmaceuticals}

1. Clinical trials are a medical approach that is used to diagnose and prevent disease. Many systems have been created in recent decades to prevent and diagnose diseases. However, these systems have some flaws, including data integrity, record-sharing, data privacy, and patient enrollment, that can solve these issues with blockchain technology. The following are a few clinical healthcare systems that provide data integrity and privacy. Healthcare is a token-based currency that is used to keep track of information on hospital employees, doctors, health plans, and insurance companies, among other things. FHIRChain (Zhang et al. 2018a, b) is a smart health system based on smart contracts that allow clinical healthcare data to be exchanged.

2. Similarly, Connecting Care (Nugent et al. 2016) is a blockchain-based record-sharing platform accessible in several English cities. In a diversified healthcare organization, Connecting Care is utilized to secure data relating to hospitals and other medical record data. It provides an access control list, ensuring that only authorized users have access to the clinical system. Ethereum-based framework is used for implementing smart contract capabilities in the blockchain. To enroll a patient in the Healthcare system, the system employs an enrolment strategy. The authorities have access to the patient's medical information, and the patient can submit their personal information to the system (Hang et al. 2019).
Clinical environments of blockchain technologies will undoubtedly open up new scientific opportunities for medical research to progress. While, on the other side, the reliable, safe and scalable collection, storing, and retrieving of these medical studies in precision medicine applications will help establish compelling opportunities for illness identification and treatment. For cognitive schemes, a database might be used. A digitized brain could be processed on a blockchain, and neurotechnologies are at a somewhat experimental stage. Only a few enterprises have gone so far as to announce a position for blockchain technology (Brandon et al. 1991).

\section{Blockchain in Healthcare industry}

In 2016, blockchain was a hot subject, while the system has been in operation since 2009 and is one of the primary components used to exchange the Bitcoin digital currency (Zhou et al. 2018). The system gets a lot of healthcare consideration and funding from the office of the National Health Information Technology manager because of its potential capacity to address many fundamental healthcare problems as this technology is used to control and transport electronic health records, confidentiality, compatibility, standardized shared infrastructure, and international standards would become accessible with the ever-changing technological world and relentless healthcare advancement, IT executives recognize that their goal is to implement the correct health IT trends and innovations (Dubovitskaya et al. 2017b). However, health IT administrators have to be cautious not to avoid becoming adopters of emerging technologies that place a patient's wellbeing at risk (Taylor 2016).

The pharma industry is one of the fastest-growing sectors globally. It is a critical player in patient outcomes aids in the delivery of experimental and potentially beneficial treatments to the market. It maintains the quality and efficacy of prescription devices and drugs marketed to the general public. Furthermore, it assists in diagnosing and processing sterile medications, resulting in faster patient recovery (Haq and Muselemu 2018). In most cases, drug makers face difficulties monitoring their goods promptly, which may pose severe risks by encouraging counterfeit goods to compromise manufacturing or infiltrate the counterfeit medications system. As a result, its production and distribution have become a significant global health threat, especially in developed countries.

Blockchain may be a best-fit technology for testing, tracking, and ensuring the manufacturing processes of potential medicines during the production and research and development (R\&D) of these drugs (Jamil et al. 2020). An automated drug management scheme (DDCS) may be a long-term solution for preventing illicit drugs in this regard. Big pharmaceutical companies (Sanofi, Pfizer, and Amgen) initiated a collaborative pilot project to inspect and assess experimental medicines using a blockchain-based DDCS (Casado-Vara et al. 2018a). 
Blockchain is essentially a distributed ledger (database) that can be programmed to record online financial transactions in such a way that they are secure and cannot be manipulated. In the blockchain, each transaction is digitally signed from participants to ensure its authenticity and security. The distributed ledger operates by consensus (smart contracts). Both parties agree at the ledger to put each transaction into a block and validate that block to add it to a chain. Each block contains data and information (Khan et al. 2020a, b). Finally, that chain is protected using cryptography algorithms, and consequently, it cannot be manipulated or changed. Blockchain is a decentralized technology that ensures the security of data, and no one can manipulate transaction data because of its many replicas in different servers. Data resources stored in centralized servers are vulnerable to cybercrime. On the other hand, blockchain ensures the security and privacy of data by storing it in decentralized locations (Khan et al. 2020a, b). Over the past few years, the market value of blockchain technology increased substantially. Blockchain technology is more trusted and secure than all other record-keeping systems. It is a distributed ledger where all nodes of the network share the same documentation. It is also used to increase efficiency and speed by automating the traditional process with blockchain technology. It also reduces costs because it does not require the buying and selling of products through third parties.

\section{Immutability}

The blockchain data structure is built by time stamping a ledger, connecting it, and accessing it with the previous documents. The blockchain's decentralized existence allows it to store every form of record, such as healthcare, banking, and supply chains, wherever authenticity, authenticity, and audit capability are essential (González et al. 2015; Schechtman 2019).

\section{Decentralized consensus}

The strength of a blockchain derives from decentralizing it. Cryptography and immutability are the keys to being adulterated by the blockchain. It is a decentralized agreement, though, that implements it. This organization will change a blockchain where a single entity or organization operates all networks. All it needs is the blockchain nodes that want to authorize the operation (O'Hara 2017). However, it is far tougher to undermine the blockchain's consistency because several parties with varying incentives must settle on what constitutes a legal transaction by decentralized consensus. In this scenario, many organizations operating a few nodes each is the most efficient model because it is incredibly doubtful that more than $50 \%$ of the nodes would intentionally consent to compromise the blockchain data (Nakamoto 2018).

\section{Baked-in incentives}

A blockchain turns networks into markets at a high stage; an individual of the network needs to do particular work, such as saving data and validating transactions. The node provides some profit in proportion to the work done to compensate for that task and is a distributed ledger system that offers a safe, decentralized, distributed information store tamperproofed (Ilinca 2020). Blockchain offers a trustless mechanism that creates a network of open networks where a community of non-trusting individuals can cooperate and conduct business without approval from third parties and provides a shared data archive, where any peer in the network holds a copy of mirrored data, rather than hierarchical records management of transactions processing (De Filippi et al. 2020).

The network handles the replication, exchange, and synchronization of the peers' data by any consensus process. The digital currency is designed on top of a series of interaction protocols that enable the shared management of transactions through many computing devices that are geographically isolated (Jennath et al. 2020).

Blockchain e-Health is a concept commonly used in the healthcare industry that has been emerging since 2000 to apply to information and communication technology (ICT). It can, across the Internet and associated technology, supply creative solutions in the medical sector covering applications such as medical informatics, patient healthcare, health care practitioners, health care personnel, medicine, etc., relating to clinical insurance, and health details. If the accessibility of knowledge or data becomes seamless through expanded Software and Applications and mobile devices, patients gain access to their medical history and details regarding care (Yue et al. 2016).

\section{Securing e-Health system and efficient data}

The storage and management of medical details need a consistent concept of access control, authentication, and immutability of data information to ensure the integrity, confidentiality, and reliability of health documents and health reports that contain all wearable sensor results and service facilities' patient treatment records (Dwivedi et al. 2019). Due to data sensitivity, as medical record-keeping involves privacy and confidentiality, role-based consent for data upload and also data processing should be enforced. Auditing processes can be applied to record and document the requests and access the data to be tracked. With a protected data access management mechanism for multi-party enterprises, blockchain offers an efficient structure for building a private/public network. The ability of blockchain to build a stable, decentralized network for data exchange has recently been investigated. A methodology for using a private blockchain for medical record booking and the Healthcare Data Gateway (HDG) introduction has 
been identified to encourage customers to maintain their healthcare records ( $\mathrm{Li}$ and Han 2019).

Casado-Vara et al. suggested introducing personal medical records management in the digital blockchain, where patient documents are authenticated and maintained publicly in a distributed manner (Casado-Vara et al. 2018b). Blockchain technology, though, has its share of consequences regarding the location of blockchain data information (Gole and Lakshminarayana 2020). There are, therefore, few attempts to use blockchain as an instigator for trustworthy healthcare networks. They did not accept blockchain as a data layer but used digital currencies and blockchain to enable the processing or governance of the knowledge. Blockchain-based decentralized user authentication was to monitor and handle encrypted off-chain info (Zhang et al. 2018a, b). FHIRChain is a healthcare usage scenario and is a health data-sharing mechanism operated through a smart contract where accurate clinical data does not exist in the blockchain (Azaria et al. 2016). However, the technology comes with a number of challenges that have slowed deployment, including scalability, restricted interoperability, a scarcity of blockchain developers, insufficient standards, high energy consumption, and a lack of regulatory certainty (Agbo et al. 2019). There are core priorities in deploying secure blockchain-based EHR systems along with the challenges with blockchain adoption which have been mentioned in Table 1

\section{Artificial intelligence in e-Health}

Artificial intelligence (AI) is characterized as the science and engineering of developing smart computer systems that can execute occupational stress from humans without seeking advice (Paul et al. 2021). To provide an answer or respond to a query, these computer systems use a variety of proposed algorithms and decision-making capability, as well as large quantities of data. We are surrounded by technologies capable of supporting us in our everyday lives. The performance of automated vehicles, developments in health studies, and personal wearable technology have shown technology's immense ability and how far it has advanced in recent decades (Wahl et al. 2018). Healthcare is likely to remain the first industry where artificial intelligence can have an effect beyond usability and positively impacts individual lives, considering the strides that many other sectors have created (Jiang et al. 2017).

AI can detect indicators of Alzheimer's disease in a patient's brain scans before clinicians can, according to a recent study published in Neurobiology of Aging, and is being used to do comparative studies on scans of healthy brains and those affected by Alzheimer's disease (Mathotaarachchi et al. 2017). According to a recent paper by the University of Tokyo, Watson, IBM's intellectual supercomputer, accurately detected a rare form of leukemia in a 60 -year-old lady. Watson also advised a specific treatment plan for her recovery. Watson devised a particular program for oncologists, in which it supports clinical analysts in determining treatment alternatives based on clues. Medical Sieve, a tool designed to aid clinical decision-making in cardiology and radiology, was also developed (Abujamous et al. 2018).

\section{Features of artificial intelligence in e-Health}

There are various other technologies within healthcare where AI can provide a tremendous benefit, illustrated in Fig. 2. Still, health organizations ought to analyze whether any or any of them can be implemented to continue their journey in the AI room. These are fields where steam is gaining in artificial intelligence in healthcare (Hashimoto et al. 2018).

\section{Virtual advisor for personal health}

They are expected to access an insightful private virtual assistant on their phones, with most of today's US teenagers, adults, and seniors having a mobile (Hashimoto et al. 2018). Robust systems with powerful AI technologies enable the likes of Cortana and Siri. When paired with healthcare applications, these structures can have immense value (Wahl et al. 2018). Healthcare applications may be utilized to assess the present emotional status, including drug warnings, patient education materials, and human-like experiences (Hasan Sapci and Aylin Sapci 2019). When health personnel is not present, the use of $\mathrm{AI}$ in the context of an executive secretary will have an enormous effect on tracking and supporting patients with any of their needs (Vopham et al. 2018).

\section{Advanced data analysis and screening}

AI's skills do not end at understanding human orders and recognizing what kind of answer is needed. In several specialized cases of usage in oncology, for instance, AI has been used to help identify anomalies in X-rays and magnetic resonance imaging (MRI), in genomics to conduct sophisticated sequencing, and in precision medicine to help develop highly personalized therapies for particular patients (Lucas et al. 2014).

The AI has effectively extended its capability to process organized and unstructured patient information in the IBM Watson example. In oncology, IBM Watson would provide cancer patients with proof guidelines for the medication (Zou et al. 2020).

\section{Personal life coach}

The significance of keeping communication with their patients from outside the examination room is understood by 
Table 1 Challenges with blockchain adoption and core priorities in deploying secure blockchain-based EHR systems

\begin{tabular}{|c|c|}
\hline Challenges & Bugs in the security system \\
\hline Security & $\begin{array}{l}\text { Malicious software can use security flaws to create } \\
\text { decentralized applications based on the built } \\
\text { blockchain. }\end{array}$ \\
\hline & $\begin{array}{l}\text { These malicious attacks take advantage of security flaws } \\
\text { in smart contract implementation to aid other crimes } \\
\text { like identity theft and data theft. }\end{array}$ \\
\hline
\end{tabular}

Privacy

The main challenge in protecting patient data privacy is to present a framework for data privacy and integrity on a blockchain-based EHR that leverages cryptographic methods.

This feature makes it impossible to identify a specific patient based on his current account number. In any similar system should rectify the flaws in the protection of patient data.

For starters, patients should easily exchange their data because employing blockchain-based frameworks within EHR demands a lot of computer power and takes a long time to finish each task.

Second, adding a new node to the blockchain network, which new patients require, necessitates a series of measures to ensure that the patient is trustworthy.

Scalability Lack of scalability is another challenge and higher overhead or computational resources in IoMT devices.

As a result of this challenge, the blockchain infrastructure's total processing requirements may be increased.

When a large number of smart devices or sensors are present, the problem becomes even more challenging because these devices' computing capabilities are less than that of a typical computer.

The IoT devices in the blockchain network are computationally demanding and include a large overhead bandwidth, resulting in data delays and significant processing power.

Such devices may lack the computing capacity required to employ blockchain features, forcing them to function at suboptimal or potentially exorbitant speeds, prohibiting them from simultaneously running their original and blockchain software.

Interoperability The capacity to transmit, analyze, and deal with the allocation across different blockchain networks without the use of an intermediary or central authority is referred to as blockchain interoperability. Because of the absence of interoperability, mass adoption may be nearly impossible.
Core priorities in deploying secure blockchain

References

Three goals must fulfill in terms of the following:

1. Confidentiality: Only authorized users have access to the information.

2. Integrity: data must be correct when in transit and must not be tampered with by an illegitimate group.

3. Availability: Access to information and services for legal users is not unfairly withheld.

The following requirements must be met for public blockchain privacy protection in healthcare applications: (1). Links among transactions should not be accessible or visible.

(2). The information of transaction patterns should be revealed only to their participants. However, a healthcare application built on a private or consortium blockchain can set up an access control policy to meet the data security requirements. (3). The privacy protection of transactions in a public blockchain setting is a "double-edged sword." A well-behaved patient, on the one hand, wants to keep his identity and actions confidential.

(4). On the other hand, an opposing party may use the privacy protection mechanism to conduct an illicit transaction. From the standpoints of legal traceability and accountability, the security of blockchain transactions in healthcare applications could be constrained so that the authority is trustworthy.

(5). Researchers should look into how to monitor a particular user and collect all of the messages he has sent out while keeping the user's critical information private.

(6). One potential research problem is to improve privacy in a blockchain with untrustworthy ambient assumptions and low processing costs from a development standpoint.

(7). Secure multiparty computation is a potential approach for allowing an untrusted third party to do calculations on patient data without infringing on their privacy.

As medical data grows, research is being done on the scalability of blockchain in healthcare applications.

(Al et al. 2019)

Researchers have seen an increase in interoperability efforts to bridge the gap between different blockchains. Many of them try to link private networks to public blockchains or vice versa. Prior approaches that concentrated on public blockchains and cryptocurrency-related tools were less valuable to corporate executives in the long run.
(Liang et al. 2018)

(Liang et al. 2018) 
Table 1 (continued)

\begin{tabular}{|c|c|c|c|}
\hline Challenges & Bugs in the security system & Core priorities in deploying secure blockchain & References \\
\hline & $\begin{array}{l}\text { Existing EHR solutions rely on centralized local } \\
\text { databases and offline architecture, whereas blockchain } \\
\text { technology is decentralized and cloud-based. } \\
\text { Moving healthcare systems in this direction and } \\
\text { integrating blockchain technology will necessitate the } \\
\text { development of an effective EHR system capable of } \\
\text { fostering collaboration and interoperability across } \\
\text { medical and scientific communities. }\end{array}$ & & \\
\hline Anonymity & $\begin{array}{l}\text { As public ledgers, Bitcoin blockchain and Ethereum } \\
\text { require transactions to be visible by default. }\end{array}$ & $\begin{array}{l}\text { The Ethereum network provides pseudo-anonymity; } \\
\text { transactions, for example, are connected to addresses } \\
\text { that correspond to public keys derived from user-held } \\
\text { private keys, rather than usernames or passwords. } \\
\text { Public Ethereum, also known as zk-SNARKS } \\
\text { (zero-knowledge succinct non-interactive argument of } \\
\text { knowledge), is a cryptographic proof mechanism that } \\
\text { allows a user to verify a transaction without exposing } \\
\text { the transaction's underlying data or engaging with the } \\
\text { user who broadcast it. } \\
\text { In the context of a blockchain, zk-SNARKs allow users to } \\
\text { keep their transactions private while still verifying } \\
\text { them according to the consensus process of the } \\
\text { network. } \\
\text { Once implemented, businesses will be able to transact in } \\
\text { total anonymity on the same network as their } \\
\text { competitors while benefiting from the security of the } \\
\text { public Ethereum blockchain. }\end{array}$ & $\begin{array}{l}\text { (Liang et al. } \\
\text { 2018) }\end{array}$ \\
\hline Latency & $\begin{array}{l}\text { Blockchain technology will take time to gain consensus } \\
\text { and confirm transactions, which could be an issue } \\
\text { when integrating blockchains into healthcare } \\
\text { applications that require real-time responses to events } \\
\text { and data. A blockchain takes time to process } \\
\text { transactions, which is known as transaction latency. } \\
\text { The bitcoin blockchain, for example, has a delay of } \\
10 \text { min to confirm each transaction in the network. } \\
\text { Although five or six blocks must be added to the chain } \\
\text { before confirmation, it is recommended that each } \\
\text { transaction be confirmed within } 1 \text { h. On the other hand, } \\
\text { most traditional database systems only take a few } \\
\text { seconds to confirm a transaction. }\end{array}$ & $\begin{array}{l}\text { Lower latency has been linked to blockchain-based IoT } \\
\text { devices, but they can be applied to other blockchain } \\
\text { applications. } \\
\text { The IoT network, which has a large number of devices } \\
\text { communicating with each other at the same time, } \\
\text { necessitates a network with latency. } \\
\text { The consensus method confirms each block's transaction, } \\
\text { which significantly reduces latency affecting the } \\
\text { application's overall performance. }\end{array}$ & $\begin{array}{l}\text { (Badr et al. } \\
\text { 2018) }\end{array}$ \\
\hline
\end{tabular}

medical professionals that manage patients with chronic diseases (Bzdok and Meyer-Lindenberg 2018). As part of their overall coverage, many clinics have launched life-coaching systems. Still, the expense of such facilities makes it hard to maintain such systems compared to the existing declining reimbursements (Battineni et al. 2019). However, patients will obtain input on various data components collected on their phone or connected electronics with today's comprehensive AI capabilities and Smartphone applications (Khan and Alotaibi 2020). As a personal life coach, AI produces a personalized environment for and particular customer, whether it applies to drug adherence or is merely a positive voice that promotes wellness routines and healthier behaviors and delivers constructive reminders that can be sent back to clinicians (Burki 2019).

\section{Bots in healthcare}

One of the latest AI fields that are continuing to gain acceptance is in the sector of patient support, and as part of what healthcare companies provide, healthcare bots are expected to be accessible shortly, and a bot is an AI program that patients may communicate with to obtain support with their demands via a chat window on a website or by mobile (Patel and Kore 2020).

In chatbot counselling, another use of artificial intelligence is that the dependence on mental healthcare chatbots does not have the reciprocity and transparency of treatment that should occur in the partnership between the mental healthcare patients and the healthcare professional (be it a conversation or psychotherapist) (Patcas et al. 2019). 


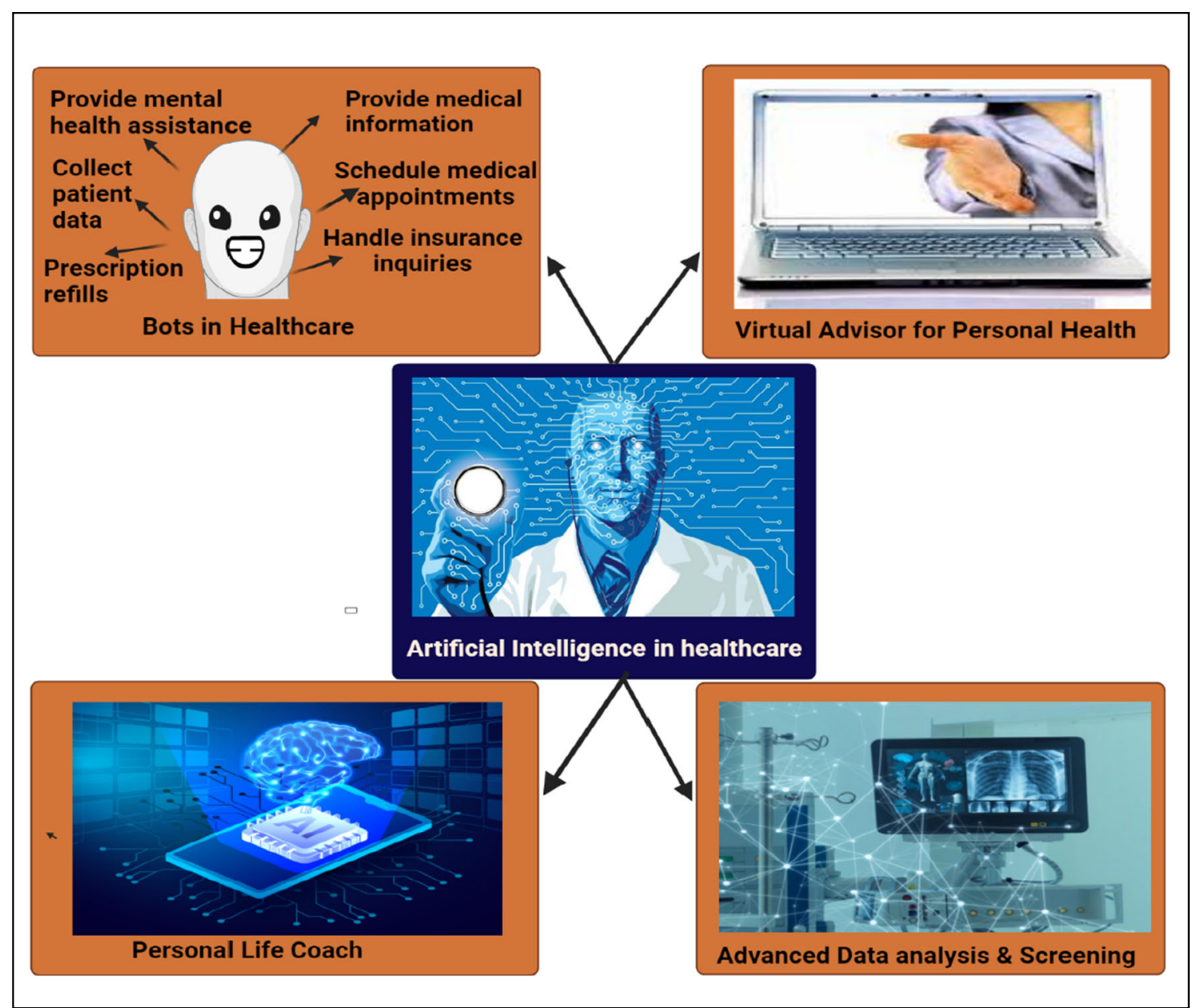

Fig. 2 Features of artificial security in e-Health

In cases such as arranging follow-up meetings with the physician of a patient online, bots may be used such cases involve whether a bots aids a customer with their criteria for treatment or billing (Adiwardana et al. 2020). Such usage cases boost patient service; provide 24/7 assistance with basic applications, such as planning, accounting, and other therapeutic applications; and minimize hospital total operating costs (George 2017). For several healthcare providers, AI, living collaboratively trained practitioners, is likely to persist in being the current course for some time, and demonstrates that AI has what it takes to diagnose a patient appropriately, the development of its current use throughout healthcare is probable to be a careful and relatively healthy framework (Han et al. 2020).

\section{Security and privacy}

Patient health information is covered by federal legislation. There can be civil and financial sanctions for any infringement or inability to uphold its credibility; as AI used by medical treatment will need access to several sets of health records, it would need to comply with the same regulations that would be followed for current applications and infrastructural facilities (Nakamoto 2018). As most AI platforms are centralized and need extensive processing resources, patient details will reside in the provider's data centers or portions. This would cause data protection issues and severe damage if the system was violated (Ilinca 2020).

Healthcare AI critics have claimed that machines are not necessarily correct and will disappoint us from time to time and when AI recommends the wrong drug or offers the wrong diagnosis to a patient, these mistakes will lead to disastrous consequences though, will ultimately progress to a point where, after it has proved its protection and preparation for medical treatment, it can be trusted. The platform will be ready to assume an active position in health treatment if its error margins are fewer than or comparable to its human peers (Ilinca 2020). At a fast pace, AI technology is evolving. Many well-known scientists and famous personalities, such as Stephen Hawking, Bill Gates, and Elon Musk, have already said that AI would be so brawny and self-conscious that it could position its interests before human interests. However, there are immense advantages of artificial intelligence in health care before robotics becomes the enemy, and several 
doctors embrace the technology. Healthcare AI provides the chance to help doctors find new choices for therapy, diagnose cancer early, and engage patients (Ahuja 2019).

\section{Al-based various tools used in healthcare}

AI, which includes machine learning, is a branch of science that employs computer algorithms to learn from data, discover patterns, and anticipate outcomes. AI and machine learning ability to analyze large and complex data structural elements to create a predictive model that personalizes and improves diagnosis, prognosis, monitoring, and treatment administration to improve individual health outcomes are among the most exciting aspects of these technologies. For decades, prediction models to aid clinical decisionmaking have existed, and these include well-known instruments illustrated in Table 2.

\section{Framingham risk score}

The Framingham risk score is among the scoring systems used to assess a person's risk of acquiring cardiovascular disease. It can find a variety of these scoring systems on the Internet (Nakhaie et al. 2018). Cardiovascular risk score systems predict the likelihood of a person developing cardiovascular disease within a given time frame, usually 10 to 30 years(Damen et al. 2019). They suggest who is most likely to benefit from prevention since they indicate the risk of acquiring cardiovascular disease. As a result, cardiovascular risk scores decide who should be prescribed preventive medications such as blood pressure and cholesterol-lowering therapies. For example, blood pressure values that surpassed high usual (130/85) were solely responsible for roughly $30 \%$ of coronary heart disease (CHD) occurrences in both men and women, demonstrating the importance of blood pressure management and monitoring for cardiovascular health and outcome prediction.

\section{QRISK$^{\oplus 3}$}

The QRISK®3 algorithm is a validated risk-stratification tool that determines a person's chances of having a heart attack or stroke in the next 10 years. It displays the average risk of persons who share the same risk variables like the person (Ho et al. 2021). The QRISK®3 algorithm was created by doctors and academics working in the UK National Health Service and is based on data collected regularly from hundreds of Global Positioning System (GPS) around the country who have willingly submitted data to the Q Research database for medical study. A patient must consult with their doctor before making any medical decisions. The authors and sponsors take no responsibility for the score's therapeutic usage or misuse. QRISK ${ }^{\circledR} 2$ is adequate for a transition time, but QRISK ${ }^{\circledR} 3$ is superior (Hippisley-Cox et al. 2017). QRISK3 derivation
Table 2 AI-based various tools used in healthcare

\begin{tabular}{|c|c|c|c|}
\hline Algorithm & Use & Interventions & References \\
\hline $\begin{array}{l}\text { Framingham } \\
\text { risk score }\end{array}$ & $\begin{array}{l}\text { It is a } \\
\text { gender-specific } \\
\text { algorithm and } \\
\text { used to estimate } \\
\text { the 10-year } \\
\text { cardiovascular } \\
\text { risk of an } \\
\text { individual. }\end{array}$ & $\begin{array}{l}\text { Individuals with low } \\
\text { risk have } 10 \% \text { or } \\
\text { less CHD risk at } 10 \\
\text { years, with } \\
\text { intermediate risk } \\
10-20 \% \text {, and with } \\
\text { high risk } 20 \% \text { or } \\
\text { more. }\end{array}$ & $\begin{array}{l}\text { (Nakhaie } \\
\text { et al. } \\
\text { 2018). } \\
\text { (Damen } \\
\text { et al. } \\
\text { 2019) }\end{array}$ \\
\hline QRISK3 & $\begin{array}{l}\text { Prediction } \\
\text { algorithm and } \\
\text { use for } \\
\text { cardiovascular } \\
\text { disease (CVD) }\end{array}$ & $\begin{array}{l}\text { QRISK3 combines } \\
\text { traditional risk } \\
\text { factors such as age, } \\
\text { systolic blood } \\
\text { pressure, smoking } \\
\text { status, and total } \\
\text { serum cholesterol to } \\
\text { elevated lipoprotein } \\
\text { cholesterol ratio } \\
\text { with body mass } \\
\text { index, ethnicity, } \\
\text { deprivation } \\
\text { measures, family } \\
\text { history, chronic } \\
\text { kidney disease, } \\
\text { muscular } \\
\text { dystrophy, atrial } \\
\text { fibrillation, diabetes } \\
\text { mellitus, and } \\
\text { antihypertensive } \\
\text { treatment. }\end{array}$ & $\begin{array}{c}\text { (Ho et al. } \\
\text { 2021) } \\
\text { (Laight } \\
2018) .\end{array}$ \\
\hline MELD score & $\begin{array}{l}\text { It is a } \\
\text { prospectively } \\
\text { developed and } \\
\text { validated } \\
\text { chronic liver } \\
\text { disease severity } \\
\text { scoring system }\end{array}$ & $\begin{array}{l}\text { MELD score uses a } \\
\text { patient's laboratory } \\
\text { values for serum } \\
\text { bilirubin, serum } \\
\text { creatinine, and the } \\
\text { international } \\
\text { normalized ratio } \\
\text { (INR) for } \\
\text { prothrombin time to } \\
\text { predict 3-month } \\
\text { survival. } \\
\text { In patients with } \\
\text { cirrhosis, an } \\
\text { increasing MELD } \\
\text { score is associated } \\
\text { with increasing } \\
\text { severity of hepatic } \\
\text { dysfunction and } \\
\text { increased 3-month } \\
\text { mortality risk. }\end{array}$ & $\begin{array}{l}\text { (Hamilton } \\
\quad \text { et al. } \\
2018 \text { ) } \\
\text { (Goldberg } \\
\text { et al. } \\
\text { 2021) }\end{array}$ \\
\hline $\begin{array}{r}\mathrm{ABCD}^{2} \\
\text { score }\end{array}$ & $\begin{array}{l}\text { Predict the risk of } \\
\text { recurrent stroke } \\
\text { soon after a } \\
\text { transient } \\
\text { ischemic attack } \\
\text { (TIA). }\end{array}$ & $\begin{array}{l}\mathrm{ABCD}^{2} \text { is increasingly } \\
\text { being used to } \\
\text { stratify referrals to } \\
\text { fast-track clinics. } \\
\text { Patients with a low } \\
\mathrm{ABCD}^{2} \text { score } \\
\text { referred to } \\
\text { cerebrovascular } \\
\text { services are more } \\
\text { prone to have a } \\
\text { noncerebrovascular } \\
\text { diagnosis than those } \\
\text { with a higher score. }\end{array}$ & $\begin{array}{l}\text { (Galvin } \\
\text { et al. } \\
2011 \text { \& } \\
\text { Uchiya- } \\
\text { ma et al. } \\
\text { 2019) }\end{array}$ \\
\hline
\end{tabular}


Table 2 (continued)

\begin{tabular}{|c|c|c|c|}
\hline Algorithm & Use & Interventions & References \\
\hline $\begin{array}{l}\text { Nottingham } \\
\text { Prognostic } \\
\text { Index }\end{array}$ & $\begin{array}{l}\text { Used to determine } \\
\text { prognosis } \\
\text { following } \\
\text { surgery for } \\
\text { breast cancer. }\end{array}$ & $\begin{array}{l}\text { Its value is calculated } \\
\text { using three } \\
\text { pathological } \\
\text { criteria: the size of } \\
\text { the tumor, the } \\
\text { number of involved } \\
\text { lymph nodes, and } \\
\text { the grade of the } \\
\text { tumor. }\end{array}$ & $\begin{array}{l}\text { (Gray et al. } \\
\text { 2018) } \\
\text { (McInnes } \\
\text { et al. } \\
\text { 2018). }\end{array}$ \\
\hline XNAT & $\begin{array}{l}\text { Developed for } \\
\text { neuroimaging } \\
\text { research. }\end{array}$ & $\begin{array}{l}\text { XNAT anonymize any } \\
\text { datasets of medical } \\
\text { imaging data. }\end{array}$ & $\begin{array}{l}\text { (Khvastova } \\
\text { et al. } \\
\text { 2020). }\end{array}$ \\
\hline $\begin{array}{l}\text { Swiss } \\
\text { ADME }\end{array}$ & $\begin{array}{l}\text { ADME modelling } \\
\text { plays a pivotal } \\
\text { role in drug } \\
\text { discovery }\end{array}$ & $\begin{array}{l}\text { It is the first web } \\
\text { interface that } \\
\text { enables batch } \\
\text { calculations for } \\
\text { hundreds of } \\
\text { different molecules, } \\
\text { allowing efficient } \\
\text { pharmacokinetic } \\
\text { optimization as well } \\
\text { as chemical library } \\
\text { analysis. }\end{array}$ & $\begin{array}{l}\text { (Mishra and } \\
\text { Dahima } \\
\text { 2019) }\end{array}$ \\
\hline
\end{tabular}

allowed cholesterol values from after the index date to be used if they were measured before any event; instead, we only included values recorded before the index date to avoid using future information in prediction), and we evaluated the Townsend deprivation score as the median of the vigintile (equal 20th) of the score for the area within which an individual lived, as individual values were not available (Laight 2018).

\section{MELD score}

The model for end-stage liver disease (MELD) was developed to predict survival in patients with portal hypertension problems undergoing elective transjugular intrahepatic portosystemic shunt installation (Kamath et al. 2001). Given its accuracy in predicting short-term survival among patients with cirrhosis, MELD was initially adopted by the United Network for Organ Sharing (UNOS) in 2002 for prioritization of patients awaiting liver transplantation in the USA (Jung et al. 2008). The MELD, which relies solely on objective factors, was later proven as a reliable predictor of survival in various groups of patients with advanced liver disease (Yip et al. 2018). The MELD score has been chiefly used in the allocation of organs for liver transplantation (Sacleux and Samuel 2019). On the other hand, the MELD score has been proven to predict survival in cirrhotic patients with infections, hemorrhage, fulminant hepatic failure, and alcoholic hepatitis. MELD score also used to determine the best treatment for individuals with hepatocellular carcinoma who are not candidates for liver transplantation and identify patients for surgery other than liver transplantation (Hamilton et al. 2018). Despite the MELD score's many benefits, about 15 to $20 \%$ of patients' survival cannot be reliably predicted by the MELD score. Likely, adding factors that are better predictors of liver and renal function will increase the model's prediction accuracy. The MELD score will be further refined and validated in the future (Goldberg et al. 2021).

\section{$A B C D^{2}$ score}

A clinical testing instrument is used outside of a hospital setting for people at high risk of stroke after a transient ischemic attack, which recognizes the importance of diabetes in rating risk of strokes (Galvin et al. 2011 \& Uchiyama et al. 2019).

$\mathrm{ABCD}^{2}$ score calculation
A: Age ( $\geq 60$ years, 1 point);
B: Blood pressure at presentation $(\geq 140 / 90 \mathrm{mmHg}, 1$ point);
C: Clinical features (unilateral weakness, 2 points; speech disturbance without weakness, 1 point);
D: Duration of symptoms ( $\geq 60 \mathrm{~min}, 2$ points; $10-59$ min, 1 point);

Presence of diabetes ( 1 point). Total scores range from 0 (low) risks to 7 (high) risk.

\section{Nottingham Prognostic Index}

The Nottingham Prognostic Index (NPI) is a straightforward clinic-pathological prognostic tool for breast cancer risk stratification (Abubakar et al. 2021). NPI approach categorizes early and locally advanced breast cancer instances into three or more prognostic groups (TNM stages I, II, and III) (Durrani et al. 2021). NPI plays an active role in clinical practice and research, even though more advanced models have overtaken it in some applications. In clinical practice, NPI and related prognostic models help patients and clinicians decide whether or not to have adjuvant chemotherapy after surgery (Vander et al. 2017). By allocating individuals to prognostic categories and then applying survival estimates from previous cohort research, the NPI can be utilized to offer prognostic information. The NPI is beneficial in economic evaluations as well as providing predictive estimations for specific patients. Overall survival is a significant input for decision-analytic models used to assess additional costs and benefits and determine whether and how to allocate finite healthcare expenditures to new screening and management options in the economic evaluation of novel therapies (Gray et al. 2018). When clinical trials are not likely to be possible or timely in delivering robust evidence of the impact of introducing new programs or 
adjusting existing programs, decision-analytic models play a critical role in evaluating novel technologies (McInnes et al. 2018).

\section{XNAT}

The extensible neuroimaging archive toolkit (XNAT) is one of the most widely used open-source informatics platforms for imaging research, and it will accelerate the creation of AI models by providing an end-to-end development platform, enabling faster collaborations (Timón et al. 2017). It follows the same logic as clinical research investigations. Projects, subjects, and experiments are the most common data types in XNAT, and they are often arranged in a hierarchical order. There are numerous built-in data kinds, spanning from various imaging modalities to provenance and quality assurance support.

XNAT is written in Java, and plugins are built using the Gradle build system. The build Gradle file describes the basic configuration, such as the plugin version, library dependencies, and other build processes required to construct a plugin from Java source code. The build system verifies that all dependencies are obtained before the compilation process, and the plugin jar file is generated. The plugin file is automatically incorporated into the instance on upstart if placed in the appropriate location within the XNAT directory structure (Khvastova et al. 2020).

\section{Swiss ADME}

Swiss ADME is part of the Swiss Drug Design program and driven by the Swiss Institute of Bioinformatics (Daina and Zoete 2019). It estimates ADME, pharmacokinetics, druglikeness, and medicinal chemistry friendliness of a small molecule output of any computer-aided drug designing (CADD) process (Gfeller et al. 2014). In comparison to the state-of-theart of available web-based tools for ADME (Cheng et al. 2012) and pharmacokinetics (e.g., pk-CSM, and ADMET SAR ), and aside from particular notion to proficient methods (e.g., iLOGP, or the BOILED-Egg), SwissADME's strong points include, but are not limited to, multiple input methods, computation for numerous molecules, and the ability to display, save, and share results per individual (Daina et al. 2017).

BOILED-Egg is a simple method for predicting two important ADME characteristics at the same time: passive gastrointestinal absorption and blood-brain barrier (BBB) (Daina and Zoete 2016). Although this classification model is conceptually basic because it only uses two physicochemical descriptors (WLOGP and TPSA, for lipophilicity and apparent polarity, respectively), it was designed with terrific attention in terms of statistical significance and robustness (Zoete et al. 2016). The yolk (i.e., the physicochemical area for highly probable BBB permeability) and the white make up the eggshaped classification plot (i.e., the physicochemical space for passive gastrointestinal absorption). Both compartments are not mutually exclusive, and the outer grey zone represents chemicals with characteristics that suggest low absorption and brain penetration (Pires et al. 2015).

\section{Applications of $\mathrm{Al}$ in healthcare}

AI has allowance for more precision in identifying the problem in its early stages. Drug design and chemical selection can now be automated thanks to AI. Peptone predicts protein qualities and features using $\mathrm{AI}$ in conjunction with Keras and TensorFlow, allowing experts to simplify protein planning, uncover manufacturing and depiction difficulties, and discover new protein features.

Artificial intelligence is also widely used in clinical trials to convert various biological and medical care data into PC models. The models enable physicians to differentiate patients' reactions to drugs based on their characteristics, allowing for mass delivery of personalized medication and treatment.

Another area in which $\mathrm{AI}$ is gaining traction is drug research. Helix, an AI engine, responds to vocal inquiries and requests using $\mathrm{AI}$, allowing scientists to increase their efficiency, improve lab security, stay up to date on vital research subjects, and maintain track of the inventory.

\section{Radiology}

Computerized tomography (CT) and MRI scanning are researching AI in radiology to identify and treat illness in patients (Hosny et al. 2018). According to the Radiology Community of North America, the emphasis on artificial intelligence in radiology has steadily increased over the years, where they have observed development from 0 to 3 , and a total $10 \%$ of the overall publishing AI has been capable of serving well enough for identifying anomalies and measuring progress over time by imaging in oncology, two main influences in oncological wellbeing (Bai et al. 2020).

When it comes to maintaining scanners between patients, radiology personnel must be exceedingly conscientious. In this case, chest X-rays may be a better option due to the ease with which machines can be cleaned. However, reports on the use of X-ray for COVID-19 diagnosis have been mixed. Other sources identify X-ray as an insensitive test (Chen et al. 2020) , while hospitals in Spain include the method as a default aspect of their diagnostic process.

\section{Screening}

Latest developments have proposed using AI to describe and measure the result of maxillo-facial operation or cleft palate treatment in terms of facial beauty or presentation of age (Azaria et al. 2016). 
In 2018, a reported issue of the journal Annals of Oncology described that an artificial intelligence device (using a deep learning convolutionary neural network) could more effectively diagnoses skin cancer than physicians. On average, $86.6 \%$ of skin cancers were correctly diagnosed by human dermatologists from the photos, compared to $95 \%$ for the CNN unit. Researchers demonstrated an AI device focused on a Google Deep Mind algorithm worthy of exceeding medical professionals in detecting breast cancer (Zhang et al. 2018a, b).

\section{In psychology}

AI implementations are currently in a proof-of-concept phase; chatbots that mimic human actions and are tested for depression and anxiety are fields where the evidence is increasingly expanding. However, challenges involve the possibility that commercial companies create and implement several applications in suicidal behavior screening (Agbo et al. 2019).

\section{Principal care}

The main growth area for AI technology has been primary care. AI has been used in primary care to facilitate decisionmaking, statistical modeling, and market analytics. Considering the rapid developments in AI technologies, the perspective of healthcare professionals on the role of $\mathrm{AI}$ in primary care is very minimal, concentrating primarily on organizational and repetitive reporting tasks (Liang et al. 2018).

\section{Diagnosis of illness}

An essay was revealed that for a range of different disorders, such as help vector machines, machine learning, and logistic regression, many forms of AI strategies have been used. Each of these strategies is defined as having a "training purpose" such that classifications comply as much as possible with the findings (Liang et al. 2018). There are two different methods to detect these diseases, involving the usage of artificial neural networks (ANN) and Bayesian networks (BN) to show such specifics for disease diagnosis/classification. It was learned that ANN was smarter and could identify diabetes and CVD more correctly (Fan et al. 2018).

\section{Telemedicine}

The growth of telemedicine, the virtual diagnosis of clinicians, has seen the rise of potential AI applications. Through tracking their details by detectors, AI may enable them to care for patients remotely. A wearable interface may allow a person to be continuously watched and the opportunity to detect adjustments that may be less humanly differentiated. The data may be linked to other data already gathered using algorithms of artificial intelligence that warn doctors if there are any problems to be informed of (Pianykh et al. 2020). In chat-bot counseling, another use of artificial intelligence is some scholars claim that the dependence on mental healthcare chat-bots does not have the reciprocity and transparency of treatment that should occur in the partnership between the mental healthcare patients and the healthcare professional (be it a conversation or psychotherapist) (Hosny et al. 2018).

Artificial intelligence may help to take care of elderly populations as the mean age has increased due to extended life expectancy. Instruments such as culture and human sensors will detect the daily behaviors of an individual and warn a caretaker whether an irregular activity or a calculated vital is abnormal during the tech is useful; there are still conversations surrounding shortcomings of monitoring (Patcas et al. 2019). Lei Xing et al. described the trends in research and development in artificial intelligence in medicines and their indications to the future of health care (Xing et al. 2021). They mentioned the broad scope and incredible depth of potential impact that AI promises to bring; it is quite clear that medicine is on the verge of a revolution.

Omer Adir et al.(Adir et al. 2020) has reported that the use of artificial intelligence in nanomedicine design increases therapeutic efficacy by optimizing material properties based on predicted interaction with the target drug, biological fluids, immune system, vascular system, and cell membrane, as we already study by many scientists that high intratumor and interpatient heterogeneities make rational diagnostic and therapeutic platform design, as well as analysis of their output, extremely difficult; and in Table 3, we listed few more cases of artificial intelligence, its role in different cancer.

\section{Al in drug discovery}

AI can be used effectively in different parts of drug discovery, including drug design, chemical synthesis, drug screening, polypharmacology, and drug repurposing. AI can distinguish hit and lead compounds, allowing for faster therapeutic target validation and structural design optimization. Despite its benefits, AI has substantial data difficulties, including its scale, growth, diversity, and ambiguity ( Zhu 2020).

Pharmaceutical companies' drug research data sets can contain millions of molecules, and typical machine learning algorithms may not handle them. A computational model based on the quantitative structure-activity relationship (QSAR) can swiftly predict vast numbers of compounds or superficial physicochemical characteristics like log P or $\log \mathrm{D}$ (Grosdidier et al. 2011). However, these models are a long way from predicting complicated biological features like chemical efficacy and side effects. Small training sets, experimental data error in training sets, and a lack of experimental validations are all issues that QSAR-based models confront. To address these issues, recently emerging AI 
Table 3 Role of artificial intelligence in different cancers

\begin{tabular}{|c|c|c|c|c|}
\hline Cancer & Background & $\begin{array}{l}\text { Internal \& external } \\
\text { validation }\end{array}$ & Finding & Reference \\
\hline $\begin{array}{c}\text { Breast } \\
\text { can- } \\
\text { cer }\end{array}$ & $\begin{array}{l}\text { Evaluation of an AI } \\
\text { system for breast } \\
\text { cancer screening in } \\
\text { which they introduce } \\
\text { an artificial } \\
\text { intelligence }\end{array}$ & $\begin{array}{l}\text { The AI system } \\
\text { outperformed all of } \\
\text { the human readers in } \\
\text { their analysis of six } \\
\text { radiologists: the AI } \\
\text { system's area under } \\
\text { the receiver operating } \\
\text { characteristic curve } \\
\text { (AUC-ROC) was } \\
11.5 \%>\text { normal } \\
\text { radiologist's } \\
\text { AUC-ROC. }\end{array}$ & $\begin{array}{l}\text { Clinical studies to } \\
\text { increase the precision } \\
\text { and reliability of } \\
\text { breast cancer } \\
\text { screening are now } \\
\text { possible thanks to this } \\
\text { thorough evaluation } \\
\text { of the AI scheme. }\end{array}$ & $\begin{array}{l}\text { (McKinney } \\
\text { et al. 2020) }\end{array}$ \\
\hline $\begin{array}{c}\text { Prostate } \\
\text { can- } \\
\text { cer }\end{array}$ & $\begin{array}{l}\text { A blinded clinical } \\
\text { validation analysis } \\
\text { and implementation } \\
\text { of an artificial } \\
\text { intelligence } \\
\text { (AI)-based algorithm } \\
\text { in a pathology lab for } \\
\text { routine clinical use to } \\
\text { assist prostate } \\
\text { diagnosis was } \\
\text { described. }\end{array}$ & $\begin{array}{l}\text { Set of } 32 \text { prostate CNB } \\
\text { cases (selected from } \\
\text { cases occurring } \\
\text { between August } 2014 \\
\text { and January } 2018 \text { ), } \\
\text { comprising } 159 \text { parts, } \\
\text { to calibrate the } \\
\text { algorithm for } \\
\text { UPMC-specific } \\
\text { whole-slide image } \\
\text { attributes (e.g., } \\
\text { scanner and staining) } \\
\text { and to verify the } \\
\text { technical validity of } \\
\text { the whole-slide } \\
\text { images (e.g., file } \\
\text { format and } \\
\text { resolution). Internal } \\
\text { test:benign vs cancer } \\
\text { AUC was } 0 \cdot 997, \\
\text { specificity: } 90 \cdot 14 \% \\
\text { sentivity to be found }\end{array}$ & $\begin{array}{l}\text { An AI-based algorithm } \\
\text { for accurately } \\
\text { detecting, grading, } \\
\text { and evaluating } \\
\text { clinically relevant } \\
\text { findings in digitized } \\
\text { slides of prostate } \\
\text { CNBs was developed, } \\
\text { externally clinically } \\
\text { validated, and } \\
\text { deployed in clinical } \\
\text { practice. }\end{array}$ & $\begin{array}{r}\text { (Pantanowitz } \\
\text { et al. 2020) }\end{array}$ \\
\hline $\begin{array}{l}\text { Lung } \\
\text { can- } \\
\text { cer }\end{array}$ & $\begin{array}{l}\text { End-to-end design is } \\
\text { particularly important } \\
\text { when considering AI } \\
\text { application in lung } \\
\text { cancer }\end{array}$ & $\begin{array}{l}\text { In the test dataset } \\
\text { consisting of } 6716 \\
\text { cases ( } 86 \\
\text { cancer-positives) } \\
\text { from the National } \\
\text { Lung Screening Trial } \\
\text { (NLST), this model } \\
\text { achieved an AUC of } \\
94.4 \% \text { for lung } \\
\text { cancer-risk prediction, } \\
\text { which is considered to } \\
\text { be a state-of-the-art } \\
\text { performance. The } \\
\text { model performed } \\
\text { similarly with an } \\
\text { AUC of } 95.5 \% \text { on an } \\
\text { independent clinical } \\
\text { validation set of } 1139 \\
\text { cases. }\end{array}$ & $\begin{array}{l}\text { Demonstrating a step } \\
\text { toward automated } \\
\text { image evaluation for } \\
\text { lung cancer risk } \\
\text { estimation using AI. }\end{array}$ & $\begin{array}{l}\text { (Ardila et al. } \\
\text { 2019) }\end{array}$ \\
\hline
\end{tabular}

technologies, such as deep learning (DL) and relevant modeling studies, can be used to evaluate the safety and efficacy of therapeutic compounds using big data modeling and analysis. Merck sponsored a QSAR ML competition in 2012 to investigate the benefits of DL in the pharmaceutical industry's drug discovery process. For 15 absorption, distribution, metabolism, excretion, and toxicity (ADMET) data sets of drug candidates, DL models outperformed classic ML approaches in terms of predictability (Ciallella and Zhu 2019). 
QSAR modeling methods, such as linear discriminant analysis (LDA), support vector machines (SVMs), random forest (RF), and decision trees, have been used to identify prospective drug candidates and have evolved into AI-based QSAR approaches can use that to speed up QSAR analysis (Zhang 2017 \& Jain 2015 ). The different AI tools used in drug discovery are listed in Table 4.

\section{Blockchain and $\mathrm{Al}$ integration in healthcare}

AI and blockchain flaws can be efficiently addressed by merging the two technological ecosystems (Campbell 2018). To learn, interpret, and make conclusions, AI algorithms rely on data or information. When data is acquired from a data repository or a dependable, safe, trusted, and credible platform, machine learning algorithms perform better. Blockchain acts as a distributed ledger on which data can be stored and exchanged in a cryptographically secure, validated, and agreedupon manner by all mining nodes. Data on the blockchain is stored with a high level of integrity and robustness, and it cannot be tampered with. When smart contracts are utilized to make judgments and do analytics using machine-learning algorithms, the results may be trusted and unquestioned.

Combining AI and blockchain can result in a safe, immutable, decentralized system for the sensitive data that AI-driven techniques must collect, store, and use (Marwala and Xing 2018). This approach leads to primary data and information security advances in various industries, including medical, personal, banking and financial, trading, and legal data (Mamoshina et al. 2018). Some of the key benefits of using blockchain for AI is illustrated in Table 5. Such integration allows intelligent decentralized autonomous agents (or DAOs) to validate data, value, and asset transfers among numerous authorities automatically and quickly (Magazzeni et al. 2017).
AI aids in the identification of patterns in the incoming data. Apart from facilitating more effective operations and providing insights into the patient's lifestyle and health, AI may also assist in the strategic use of analytics. AI is accomplished by studying algorithms and making tough decisions. When a patient presents to a hospital with a life-threatening illness, the computer makes the diagnosis and recommends treatment options, as well as the length of stay. Because the computer examines a large amount of data, it will be accurate. It will primarily leverage Google's vast computational power. Hanover, a Microsoft AI technology, mines data in the healthcare field using artificial intelligence. This technology allows machines to memorize medical study papers to locate potential remedies for a variety of patients. It will examine the patient's personal information by reviewing their health history, correlates it with data from medical research publications, and makes therapy recommendations (Bohr and Memarzadeh 2020). Pharmaceutical companies use blockchain technology to determine and ensure the safety and accountability of products they sell. It ensures that the data is recorded as it happens, and this is then shared analogously to deliver improved patient outcomes (Khezr et al. 2019). According to WHO estimates, around one million people die each year as a result of counterfeit drugs. Using blockchain technology, pharmaceutical corporations such as Big Pharma can verify that no counterfeit drugs exist by assigning a unique serial number to each drug (Edwin 2017). Any drug with an incorrect number would report as soon as it enters the system. This can also ensure that pharmaceuticals are not exchanged, shipped at the wrong time, or are poorly produced, among other things. Drugs are tracked using unique serialization identifiers in the following manner: National Drug Code + Unique Serial Numbers. Under the DSCSA legislation, each pack of tablets, vials, syringes, etc., has an individual unique serial number to identify the drug, where, and created. Big Pharma benefited from blockchain's full traceability of medications (Peter et al. 2017).

Table 4 AI tools used in drug discovery

\begin{tabular}{|c|c|c|}
\hline Tools & Details & References \\
\hline Organic & $\begin{array}{l}\text { A molecular design tool that aids in the } \\
\text { synthesis of molecules with specific properties. }\end{array}$ & (Brown 2015) \\
\hline DeepNeuralNet QSAR & $\begin{array}{l}\text { A python-based system that uses computational } \\
\text { methods to aid in the detection of compound } \\
\text { molecular activity. }\end{array}$ & (Chan 2019) \\
\hline Potential Net & Use to predict binding affinity of ligands & ( Pereira 2016) \\
\hline DeltaVina & Rescoring drug-ligand binding affinity with a scoring function & (Wang and Zhang 2017) \\
\hline Neural graph fingerprint & It aids in the prediction of novel molecular characteristics. & \\
\hline DeepChem & $\begin{array}{l}\text { Machine-learning model for drug discovery that employs } \\
\text { a python-based AI system to find a suitable candidate }\end{array}$ & (Arora and Bist 2020). \\
\hline DeepTox & A computer program that forecasts the toxicity of over 12,000 drugs. & (Mayr et al. 2016) \\
\hline Hit Dexter & $\begin{array}{l}\text { A machine-learning technique is being used to } \\
\text { predict which molecules will respond to a biological assay. }\end{array}$ & (Ferreira and Andricopulo 2019) \\
\hline
\end{tabular}


Table 5 Key features and benefits of blockchain integration with AI

\begin{tabular}{lll}
\hline Blockchain & AI & Blockchain and AI integration benefits \\
\hline Decentralized & Centralized & Enhanced information security \\
Deterministic & Changing & Improved trust on robotic decisions \\
Immutable & Stochastic & Making decisions based on evidence \\
Date integrity & Volatile & Decentralized intelligence \\
Attacks resilent & Data, knowledge, and decision-making are all centered on data. & High efficiency \\
\hline
\end{tabular}

\section{Blockchain and Al integration in healthcare must overcome security, interoperability concerns}

While blockchain can ensure safety and integrity, adding AI capabilities to the health sector can be beneficial. At the moment, $\mathrm{AI}$ is mainly employed to detect anomalies in X-rays and $\mathrm{CT}$ scans, a task that $\mathrm{AI}$ can perform at least as well as humans and ensure a higher level of tailored therapy. Experts believe that important inventions will emerge in the future due to the enormous benefits that may realize by lowering medical expenditures and enhancing the quality of medical care. As a result, Google, Microsoft, Apple, and Amazon, as well as a slew of startups, are actively exploring AI for medical applications, intending to improve the more effective use of patients' data, diagnosis accuracy, and providing better recommendations based on evidence-based research findings, among other things. These apps come on top of advancements in robotic surgery and digital counsel delivered via smartphone apps. According to Accenture, crucial clinical health AI applications could save the US healthcare economy $\$ 150$ billion per year by 2026 . Healthcare AI must address protection and interoperability challenges in healthcare; artificial intelligence is starting to gain strength. A modern approach to incorporate technologies in health care has been created by the convergence of sophisticated algorithms, massive data sets, and efficient machines (Tran et al. 2019).

\section{Blockchain and Al integration in storage of large healthcare data}

Medical records and other information may be uploaded to the blockchain by EHR systems. If this data is processed directly in the blockchain network, the computing overhead and storage pressure will increase due to the defined and small block size. Moreover, such data will often suffer from a privacy breach (Ahuja 2019). Most critical analyses and implementations use the framework to manage vast quantities of secure original information utilizing responsible third parties (e.g., cloud computing) and blockchain for on-chain storage to overcome these issues (Jennath et al. 2020).

\section{Blockchain and Al integration in biomedical research}

Converging blockchain and next-generation artificial intelligence technologies to decentralize and accelerate biomedical research and healthcare where they showed how deep learning is a powerful AI technique that has been effectively used in medical diagnostics, cellular image analysis, chemical syntheses, drug classification, and other areas studied by Bai et al. (Bai et al. 2021) has also illustrated in their research work on deep learning has shown to be a promising tool for medication research and discovery. The MolAICal program is used in this study to generate 3D structural ligands in the 3D pocket of protein targets using a deep learning model and a traditional approach. MolAICal is primarily made up of two modules. In the first module, FDA-approved medication fragments are utilized to build a deep learning model based on WGANs. The deep learning model's produced fragments are then used to create $3 \mathrm{D}$ ligands in the protein pocket.

The drug-like compounds from the zinc database are used to train a deep learning model based on WGANs in the second module, and molecular docking is used to estimate the affinities between synthesized compounds and proteins. MolAICal's drug design functions are tested using the membrane target GCGR and the non-membrane target SARSCoV-2 Mpro. It demonstrates that MolAICal can create ligands with varying degrees of $3 \mathrm{D}$ structural resemblance to the crystal ligand of GCGR or SARS-CoV-2 Mpro. The MolAICal provides valuable drug design tools that will aid researchers in discovering and developing new potential medications.

Other study by Krittanawong et al., (Krittanawong et al. 2020) on AI and blockchain integration can accelerate by greatly increasing the availability of data for AI training and development, able to share proprietary AI algorithms for generalization, decentralizing databases of different vendors or health systems, and incentivizing solutions that improve outcomes over those that do not, the integration of blockchain with AI could advance the goal of personalized cardiovascular medicine. However, such applications are still in their early stages, and there are some reservations regarding their implementation. More research is needed in areas such as technological trust, whether rival companies would want this flow of 
data in practice, remuneration, and ethical considerations. Using blockchain as the backbone for a network of sensors coupled to AI forecasts cardiovascular disease.

To disseminate data over a decentralized network, blockchain and artificial intelligence are being used and allow organizations to make the most of new symptoms and instances for lung cancer patients without jeopardizing their privacy concerns. This method disseminates a locally learned model for big data analytics, such as lung cancer CT scans, to collaboratively build a global model. Furthermore, this innovative technology can use a decentralized network to extract new patterns for new lung cancer patients (Kumar et al. 2021).

\section{Blockchain and Al for fighting COVID-19}

COVID-19 is a groundbreaking open-access process that gives regular estimates of new infections by country and, in certain circumstances, by location. When combined with knowledge about people's movements, it is the ideal dataset for combining blockchain and AI (Prosser 2020). Blockchain and AI are able to provide viable solutions to cope with the coronavirus epidemic from various aspects (Chamola et al. 2020). In fact, blockchain and AI have been applied to other infectious epidemics, such as Ebola (Kangbai et al. 2018) where blockchain was employed to conduct real-time Ebola contact tracing, transmission pattern surveillance, and vaccine delivery and also reveals that the cryptographic feature of blockchain can help prevent unsecure data sharing between patients and health entities such as healthcare providers (Pathak et al. 2021).

In the coronavirus outbreak fighting, blockchain can actively simplify the process of fast-tracking drug trials, and recording and tracking all fundraising activities and donations, in an immutable fashion, which can support the management of outbreaks and treatment. Moreover, AI has been applied to COVID-19-like epidemics. For example, AI techniques such as neural network architectures based on Long Short-Term Memory (LSTM) were used to predict the influenza-like illness dynamics for military populations using neural networks and social media (Lopes et al. 2021).

BlueDot, a Toronto-based start-up that uses an AIenhanced surveillance gadget, appears to have been the first to notice the epidemic in Wuhan, many hours before Chinese officials and other foreign organizations and agencies (Conway et al. 2019).

The assessment of COVID-19 cases may be supported by artificial intelligence. Infravision, for example, is a start-up that uses deep learning medical imaging platforms to speed up the detection of COVID-19 cases by recognizing complex lung features (Peng et al. 2020). Besides this, blockchain technology is a one-of-a-kind shared mechanism for storing, checking, and accepting data and performing a sequence of transactions (Rosebrock 2020). It has a high degree of protection and allows for implementing patient-centered healthcare facilities, improved public health monitoring, outbreak control, and fast and efficient decision-making, and in developed settings (to prevent crippling and straining public health ability or universal health care infrastructure) and in emerging, resource-limited circumstances, a low-cost blockchain and AI-coupled self-testing and monitoring system have been suggested for handling COVID-19 pandemics (Basu et al. 2020).

Rao and Vazquez (Rao and Vazquez 2020). propose a means based on an AI algorithm that allows for the rapid detection of infected cases, as well as risk assessment and evaluation based on symptoms and indicators associated with the novel corona virus. This would be accomplished through a web-based or mobilebased poll. In addition, based on the responses, the algorithm can send warnings to clinics or telemedicine units, requesting health checkups and case confirmation.

\section{Conclusion}

Healthcare professionals will have access to the blockchain to display the medical records of the patient, and AI uses a variety of proposed algorithms and decision-making capabilities as well as large quantities of data. Thus, by integrating the latest advances of these technologies, the medical system will have improved service efficiency, reduced costs, and democratized healthcare. Blockchain enables the storage of cryptographic records, which AI needs.

Abbreviations DNA, Deoxyribonucleic acid; IoT, Internet of Things; EHR, Electronic health records; DDCS, Automated drug management scheme; ICT, Information and communication technology; HDG, Healthcare Data Gateway; IBM, International Business Machines Corporation; CT, Computerized tomography; MR, Magnetic resonance; AI, Artificial intelligence; ANN, Artificial neural networks; BN, Bayesian networks; CVD, Cardio vascular disorder; NLST, National Lung Screening Trial; AUC, Area under curve; Zk-SNARKS, Zeroknowledge succinct non-interactive argument of knowledge; R\&D, Research and development; MRI, Magnetic resonance imaging; CHD, Coronary heart disease; GPS, Global Positioning System; MELD, Model for end-stage liver disease; UNOS, United Network for Organ Sharing; NPI, Nottingham Prognostic Index; XNAT, Extensible neuroimaging archive toolkit; CADD, Computer-aided drug designing; ADME, Absorption, distribution, metabolism and excretion; BBB, Blood-brain barrier; QSAR, Quantitative structure-activity relationship; DL, Deep learning; ML, Machine learning; RF, Random forest; DAOs, Decentralized autonomous agents

Author contribution Idea: MHR and PT1; Writing draft: PT1; Review and editing; ST, TB, PT2, HC, RA, and DK. All authors read the final version of this manuscript and approved for submission.

Data availability Not applicable

\section{Declarations}

Ethical approval Not applicable 
Consent to participate Not applicable

Consent for publication Not applicable

Competing interest The authors declare no competing interests.

\section{References}

Abujamous, Lama, et al. (2018) Towards digital cancer genetic counselling.1st International Conference on Cancer Care Informatics (CCI). IEEE.

Abubakar M et al (2021) Impact of breast cancer risk factors on clinically relevant prognostic biomarkers for primary breast cancer. Breast Cancer Res Treat:1-13

Adir O, Poley M, Chen G, et al. (2020) Integrating artificial intelligence and nanotechnology for precision cancer medicine. Adv Mater 32(13): 1901989

Adiwardana D, Luong MT, So DR, et al. (2020) Towards a human-like open-domain chatbot. arXiv2001: 09977.

Agbo C, Mahmoud Q, Eklund J (2019) Blockchain technology in healthcare: a systematic review. Healthcare 7(2):56

Ahmad SS, Khan S, Kamal MA (2019) What is blockchain technology and its significance in the current healthcare system? A brief insight. Curr Pharm Des 25(12):1402-1408

Arora K, Bist AS (2020) Artificial intelligence based drug discovery techniques for covid-19 detection. Aptisi Transactions on Technopreneurship (ATT) 2(2):120-126

Al O, Abdullah et al (2019) Privacy-friendly platform for healthcare data in cloud based on blockchain environment. Futur Gener Comput Syst 95:511-521

Ahuja AS (2019) The impact of artificial intelligence in medicine on the future role of the physician. Peer J 7:e7702

Akkiraju R. et al. (2020) Characterizing machine learning processes: a maturity framework. In: Fahland D., Ghidini C., Becker J., Dumas M. (eds) Business process management. BPM 2020. Lecture Notes in Computer Science, vol 12168. Springer, Cham.

Alotaibi YK, Federico F (2017) The impact of health information technology on patient safety. Saudi Med J38 12:1173

Ardila D, Kiraly AP, Bharadwaj S, et al. (2019) End-to-end lung cancer screening with three-dimensional deep learning on low-dose chest computed tomography. Nat Med 25(6): 954-961.

Azaria A, Ekblaw A, Vieira T, Lippman A (2016) MedRec: using blockchain for medical data access and permission management. In: Proceedings - 2016 2nd International Conference on Open and Big Data, OBD 2016, pp 25-30.

Andoni $\mathrm{M}$ et al (2019) Blockchain technology in the energy sector: a systematic review of challenges and opportunities. Renew Sust Energ Rev 21(100):143-174

Bai, Qifeng, et al. (2021) MolAICal: a soft tool for 3D drug design of protein targets by artificial intelligence and classical algorithm. Briefings in bioinformatics 22 (3): bbaal61.

Bai HX, Hsieh B, Xiong Z, Halsey K, Choi JW, Tran TML, Pan I, Shi LB, Wang D-C, Mei J, Jiang X-L, Zeng Q-H, Egglin TK, Hu P-F, Agarwal S, Xie F-F, Li S, Healey T, Atalay MK, Liao W-H (2020) Performance of radiologists in differentiating COVID-19 from viral pneumonia on chest CT. Radiology 296(2):E46-E54

Basu S, Phillips RS, Phillips R, Peterson LE, Landon BE (2020) Primary care practice finances in the United States amid the COVID-19 pandemic. Health Aff 39(9):1605-1614

Badr S, Gomaa I, Abd-Elrahman E (2018) Multi-tier blockchain framework for IoT-EHRs systems. Procedia Comput Sci 141:159-166
Battineni G et al (2019) Report on market analysis and preventions need to provide medications for rural patients of Italy using ICT technologies. Int J Innov Technol Explor Eng 9:5286-5289

Bohr A, \& Memarzadeh K (2020) The rise of artificial intelligence in healthcare applications. In Artificial intelligence in healthcare Academic Press:25-60.

Brown N. Royal Society of Chemistry (2015) Silico medicinal chemistry: computational methods to support drug design.

Bragazzi NL, Dai H, Damiani G, Behzadifar M, Martini M, Wu J (2020) How big data and artificial intelligence can help better manage the covid-19 pandemic. Int J Environ Res Public Health 17(9):3176

Brandon RM, Podhorzer M, Pollak TH (1991) Premiums without benefits: waste and inefficiency in the commercial health insurance industry. Int J Health Serv 21:265-283

Bryatov SR, Borodinov AA (2019) Blockchain technology in the pharmaceutical supply chain: researching a business model based on hyperledger fabric. CEUR Workshop Proceedings, In, pp 21-24

Burki T (2019) The dangers of the digital age. Lancet Digit Heal 1(2): e61-e62

Bzdok D, Meyer-Lindenberg A (2018) Machine learning for precision psychiatry: opportunities and challenges. Biol Psychiatry Cogn Neurosci Neuroimaging 3(3): 223-230.

Campbell D (2018) Combining ai and blockchain to push frontiers in healthcare. URL http://www. macadamian. com/2018/03/16/combining-ai-andblockchain-in-healthcare/, vol. online.

Campanella P, Lovato E, Marone C, Fallacara L, Mancuso A, Ricciardi W, Specchia ML (2016) The impact of electronic health records on healthcare quality: a systematic review and meta-analysis. Eur J Pub Health 26(1):60-64

Casado-Vara R, Prieto-Castrillo F, Corchado JM (2018a) A game theory approach for cooperative control to improve data quality and false data detection in WSN. Int J Robust Nonlinear Control 28(16): 5087-5102

Casado-Vara R, Prieto J, la Prieta FD, Corchado JM (2018b) How blockchain improves the supply chain: case study alimentary supply chain. Procedia Comput Sci 134:393-398

Cha Y, Erez T, Reynolds IJ, Kumar D, Ross J, Koytiger G, Kusko R, Zeskind B, Risso S, Kagan E, Papapetropoulos S, Grossman I, Laifenfeld D (2018) Drug repurposing from the perspective of pharmaceutical companies. Br J Pharmacol 175(2):168-180

Chan HS (2019) Advancing drug discovery via artificial intelligence. Trends Pharmacol. Sci. 40(8):592-604

Chamola V et al (2020) A comprehensive review of the COVID-19 pandemic and the role of IoT, drones, AI, blockchain, and 5G in managing its impact. Ieee access 8:90225-90265

Chapuis C, Roustit M, Bal G, Schwebel C, Pansu P, David-Tchouda S, Foroni L, Calop J, Timsit J-F, Allenet B, Bosson J-L, Bedouch P (2010) Automated drug dispensing system reduces medication errors in an intensive care setting. Crit Care Med 38(12):2275-2281

Cheng F, Li W, Zhou Y, Shen J, Wu Z, Liu G, Lee PW, Tang Y (2012) ADMET SAR: a comprehensive source and free tool for assessment of chemical ADMET properties. J Chem Inf Model 52:3099-3105

Chen N, Zhou M, Dong X, Qu J, Gong F, Han Y, Qiu Y, Wang J, Liu Y, Wei Y, Xia J', Yu T, Zhang X, Zhang L (2020) Epidemiological and clinical characteristics of 99 cases of 2019 novel coronavirus pneumonia in Wuhan, China : a descriptive study. Lancet 395:507-513

Chou W-H, Wang G, Kumar V, Weng Y-C (2015) Lipocalin-2 in stroke. Neuro - Open J 2:38-41

Cyran MA (2018) Blockchain as a foundation for sharing healthcare data. Blockchain Healthe Today 1:1-6

Ciallella HL, Zhu H (2019) Advancing computational toxicology in the big data era by artificial intelligence: data-driven and mechanismdriven modeling for chemical toxicity. Chem Res Toxicol 32:536547

Cong LW, He Z (2019) Blockchain disruption and smart contracts. Rev Financ Stud. 1;32(5):1754-1797. 
Conway M, Hu M, Chapman WW (2019) Recent advances in using natural language processing to address public health research questions using social media and consumergenerated data. Yearbook of medical informatics 28(01):208-217

Damen JA et al (2019) Performance of the Framingham risk models and pooled cohort equations for predicting 10-year risk of cardiovascular disease: a systematic review and meta-analysis. BMC Med 17(1):116

Daina A, Zoete V (2019) Application of the Swiss Drug Design online resources in virtual screening. Int J Mol Sci 20(18):4612

Daina A, Michielin O, Zoete V (2017) SwissADME: a free web tool to evaluate pharmacokinetics, drug-likeness and medicinal chemistry friendliness of small molecules. Sci Rep 7:42717

Daina A, Zoete V (2016) A boiled-egg to predict gastrointestinal absorption and brain penetration of small molecules. ChemMedChem 11(11):1117-1121

De Filippi P, Mannan M, Reijers W (2020) Blockchain as a confidence machine: the problem of trust \& challenges of governance. Technol Soc 62:101284

Dubovitskaya A, Xu Z, Ryu S et al (2017a) Blockchain dans la eSanté: perspectives et une application pour le traitement quotidien. Swiss Med Informatics 33:2017

Dubovitskaya A, Xu Z, Ryu S, et al. (2017b) Secure and trustable electronic medical records sharing using blockchain. AMIA. Annu Symp proceedings AMIA Symp 2017:650.

Durrani S, al-Mushawa F, Heena H, Wani T, al-Qahtani A (2021) Relationship of Oncotype Dx score with tumor grade, size, nodal status, proliferative marker Ki67 and Nottingham Prognostic Index in early breast cancer tumors in Saudi Population. Ann Diagn Pathol 51:151674

Dwivedi AD, Srivastava G, Dhar S, Singh R (2019) A decentralized privacy-preserving healthcare blockchain for IoT. Sensors (Switzerland) 19(2):326

Evans JG (1993) Hypothesis: healthy active life expectancy (HALE) as an index of effectiveness of health and social services for elderly people. Age Ageing 22(4):297-301

Edwin, Lopez (2017) Big Pharma builds blockchain prototype to stop counterfeits. Supply Chain Dive.

Fan K, Ren Y, Wang Y, Li H, Yang Y (2018) Blockchain-based efficient privacy preserving and data sharing scheme of content-centric network in 5G. IET Commun 12(5):527-532

Feingold E, Fein R (1987) Medical care, medical costs: the search for a health insurance policy. J Public Health Policy 8:587-590

Feng Q, He D, Zeadally S, Khan MK, Kumar N (2019) A survey on privacy protection in blockchain system. J Netw Comput Appl 126:45-58

Ferreira LL, Andricopulo AD (2019) ADMET modeling approaches in drug discovery. Drug Discov Today 24(5):1157-1165

Funk E, Riddell J, Ankel F, Cabrera D (2018) Blockchain technology: a data framework to improve validity, trust, and accountability of information exchange in health professions education. Acad Med 93(12):1791-1794

Galvin R, Geraghty C, Motterlini N, Dimitrov BD, Fahey T (2011) Prognostic value of the $\mathrm{ABCD} 2$ clinical prediction rule: a systematic review and meta-analysis. Fam Pract 28(4):366-376

George D (2017) What can the brain teach us about building artificial intelligence? Behav Brain Sci 40

Goldberg D, Mantero A, Newcomb C, Delgado C, Forde KA, Kaplan DE, John B, Nuchovich N, Dominguez B, Emanuel E, Reese PP (2021) Predicting survival after liver transplantation in patients with hepatocellular carcinoma using the LiTES-HCC score. J Hepatol 74(6):1398-1406

Gfeller D, Grosdidier A, Wirth M, Daina A, Michielin O, Zoete V (2014) SwissTargetPrediction: a web server for target prediction of bioactive small molecules. Nucleic Acids Res 42:W32-W38
Gole R, Lakshminarayana DC (2020) Seven level modular multilevel converter with Fft analysis. Int J Eng Appl Sci Technol 5(3):553558

González A, Ramos J, De Paz JF, Corchado JM (2015) Obtaining relevant genes by analysis of expression arrays with a multi-agent system. ADCAIJ Adv Distrib Comput Artif Intell J 3:137-146

Gray E et al (2018) Survival estimates stratified by the Nottingham Prognostic Index for early breast cancer: a systematic review and meta-analysis of observational studies. Systematic reviews 7(1):1-9

Grosdidier A, Zoete V, Michielin O (2011) SwissDock, a protein-small molecule docking web service based on EADock DSS. Nucleic Acids Res 39:W270-W277

Hamilton EC, Balogh J, Nguyen DT, Graviss EA, Heczey AA, Austin MT (2018) Liver transplantation for primary hepatic malignancies of childhood: the UNOS experience. J Pediatr Surg 53(1):163-168

Han C, Rundo L, Murao K, et al. (2020) Bridging the gap Between AI and healthcare sides: towards developing clinically relevant AIpowered diagnosis systems. In: IFIP Advances in information and communication technology; 320-333.

Hang L, Choi E, Kim DH (2019) A novel EMR integrity management based on a medical blockchain platform in hospital. Electronics 8: 467

Haq I, Muselemu O (2018) Blockchain technology in pharmaceutical industry to prevent counterfeit drugs. Int J Comput Appl 180(25): $8-12$

Hasan Sapci A, Aylin Sapci H (2019) Innovative assisted living tools, remote monitoring technologies, artificial intelligence-driven solutions, and robotic systems for aging societies: systematic review. JMIR Aging 2(2):e15429

Hashimoto DA, Rosman G, Rus D, Meireles OR (2018) Artificial intelligence in surgery: promises and perils. Ann Surg 268:70-76

Hippisley-Cox, J., Coupland, C., \& Brindle, P. (2017) Development and validation of QRISK3 risk prediction algorithms to estimate future risk of cardiovascular disease: prospective cohort study. bmj, 357.

Ho, Ying JS et al (2021) Cardiovascular risk quantification using QRISK3 score in people with intellectual disability. BJPsych Open 7(S1): S52-S53

Hosny A, Parmar C, Quackenbush J, Schwartz LH, Aerts HJWL (2018) Artificial intelligence in radiology. Nat Rev Cancer 18:500-510

Hylock RH, Zeng X (2019) A blockchain framework for patient-centered health records and exchange (healthChain): evaluation and proof-ofconcept study. J Med Internet Res 21.

Ilinca D (2020) Applying blockchain and artificial intelligence to digital health. In: Wulfovich S, Meyers A (eds) Digital health entrepreneurship. Health informatics. Springer, Cham

Jain N (2015) In silico de novo design of novel NNRTIs: a bio-molecular modelling approach. RSC Adv 5:14814-14827

Jamil F, Ahmad S, Iqbal N, Kim DH (2020) Towards a remote monitoring of patient vital signs based on IoT-based blockchain integrity management platforms in smart hospitals. Sensors, 20(8):2195

Jennath HS, Anoop VS, Asharaf S (2020) Blockchain for healthcare: securing patient data and enabling trusted artificial intelligence. Int J Interact Multimed Artif Intell 6:15-23

Jiang F, Jiang Y, Zhi H, Dong Y, Li H, Ma S, Wang Y, Dong Q, Shen H, Wang Y (2017) Artificial intelligence in healthcare: past, present and future. Stroke Vasc Neurol 2(4):230-243

Jung GE, Encke J, Schmidt J, Rahmel A (2008) Model for end-stage liver disease.Der. Chirurg 79(2):157-163

Kamath PS, Wiesner RH, Malinchoc M, Kremers W, Therneau TM, Kosberg CL, D'Amico G, Dickson ER, Kim WR (2001) A model to predict survival in patients with end-stage liver disease. Hepatology 33(2):464-470

Kangbai JB et al (2018) Tracking Ebola through cellphone, Internet of Things and blockchain technology.Curr. Res Integr Med 1(2):13-15 
Khan ZF, Alotaibi SR (2020) Applications of artificial intelligence and big data analytics in m-Health: a healthcare system perspective. J Healthc Eng 2020:1-15

Khan PW, Byun Y (2020) A blockchain-based secure image encryption scheme for the industrial Internet of Things. Entropy 22:175

Khan PW, Byun YC, Park N (2020a) A data verification system for CCTV surveillance cameras using blockchain technology in smart cities. Electronics 9:484

Khan PW, Byun YC, Park N (2020b). IoT-blockchain enabled optimized provenance system for food industry 4.0 using advanced deep learning. Sensors 20(10):2990.

Khezr S, Moniruzzaman M, Yassine A, Benlamri R (2019) Blockchain technology in healthcare: a comprehensive review and directions for future research. Appl Sci 9(9):1736

Khurshid A (2020) Applying blockchain technology to address the crisis of trust during the COVID-19 pandemic. JMIR Med Informatics 8(9):e20477

Khvastova $\mathrm{M}$ et al (2020) Towards interoperability in clinical researchenabling FHIR on the open-source research platform XNAT. J Med Syst 44(8):1-5

Krittanawong C, Rogers AJ, Aydar M, Choi E, Johnson KW, Wang Z, Narayan SM (2020) Integrating blockchain technology with artificial intelligence for cardiovascular medicine. Nat Rev Cardiol 17(1): $1-3$

Kumar R, Wang WY, Kumar J, Yang T, Khan A, Ali W, Ali I (2021) An integration of blockchain and AI for secure data sharing and detection of CT images for the hospitals. Comput Med Imaging Graph 87: 101812

Laight D (2018) Can LDL cholesterol ever be lowered too much. Prescriber 29(8):13-18

Li H, Han D (2019) EduRSS: a blockchain-based educational records secure storage and sharing scheme. IEEE Access 7:179273-179289

Liang J, Zervakis M, Penders J (2018) Guest editorial IEEE BHI 2017. IEEE J Biomed Heal Informatics 22(2)

Lin C, He D, Huang X, Khan MK, Choo KKR (2020) DCAP: a secure and efficient decentralized conditional anonymous payment system based on blockchain. IEEE Trans Inf Forensics Secur 15:2440-2452

Linn LA, Koo MB (2016) Blockchain for health data and its potential use in health IT and health care related research. ONC/NIST Use Blockchain Healthc Res Work: 1-10.

Lopes AR, Dias AS, Sá-Moura B (2021) Application of technology in healthcare: tackling COVID-19 challenge-the integration of blockchain and Internet of Things. In Political and Economic Implications of Blockchain Technology in Business and Healthcare. IGI Global:194-217

Lucas GM, Gratch J, King A, Morency LP (2014) It's only a computer: virtual humans increase willingness to disclose. Comput Hum Behav 37:94-100

Mandl KD (2001) Public standards and patients' control: how to keep electronic medical records accessible but private commentary: open approaches to electronic patient records commentary: a patient's viewpoint. BMJ322(7281): 283-287.

Marwala T, \& Xing B (2018) Blockchain and artificial intelligence. arXiv preprint arXiv: 1802.04451.

McKinney SM, Sieniek M, Godbole V et al (2020) International evaluation of an AI system for breast cancer screening. Nature 577(7788): 89-94

Mamoshina P, Ojomoko L, Yanovich Y, Ostrovski A, Botezatu A, Prikhodko P, Izumchenko E, Aliper A, Romantsov K, Zhebrak A, Ogu IO, Zhavoronkov A (2018) Converging blockchain and nextgeneration artificial intelligence technologies to decentralize and accelerate biomedical research and healthcare. Oncotarget 9(5): $5665-5690$

Mannaro K, Pinna A, Marchesi M (2017) Crypto-trading: Blockchainoriented energy market. In 2017 AEIT International Annual Conference: $1-5$. IEEE
Magazzeni D, McBurney P, Nash W (2017) Validation and verification of smart contracts: a research agenda. Computer 50(9):50-57

Mathotaarachchi S, Pascoal TA, Shin M, Benedet AL, Kang MS, Beaudry T, Fonov VS, Gauthier S, Rosa-Neto P, Alzheimer's Disease Neuroimaging Initiative (2017) Identifying incipient dementia individuals using machine learning and amyloid imaging. Neurobiol Aging 59:80-90

Mayr, A., Klambauer, G., Unterthiner, T., \& Hochreiter, S. (2016) DeepTox: toxicity prediction using deep learning. frontiers in environmental science 3: 80 .

McInnes, Elizabeth, et al. (2018) Barriers and facilitators to implementing stroke protocols in the emergency department. Process evaluation of the T3 trial. Int J Stroke: 6-6.

Mishra S, Dahima R (2019) In vitro ADME studies of TUG-891, a GPR120 inhibitor using SWISS ADME predictor. J Drug Deliv Ther 9(2-s):366-369

Nakamoto N (2018) Centralised bitcoin: a secure and high performance electronic cash system. SSRN Electron J. 3065723.

Nakhaie MR, Koor BE, Salehi SO, Karimpour F (2018) Prediction of cardiovascular disease risk using framingham risk score among office workers, Iran, 2017. Saudi J Kidney Dis Transpl 29(3):608-614

Nugent T, Upton D, Cimpoesu M (2016) Improving data transparency in clinical trials using blockchain smart contracts. F1000Research 5: 2541

Narayanaswami, Chandra, et al. (2019) Blockchain anchored supply chain automation. IBM J Res Dev: 7-1.

O’Hara K (2017) Smart Contracts - Dumb Idea. IEEE Internet Comput 21(2):97-101

Pantanowitz L, Quiroga-Garza GM, Bien L, Heled R, Laifenfeld D, Linhart C, Sandbank J, Shach AA, Shalev V, Vecsler M, Michelow P (2020) An artificial intelligence algorithm for prostate cancer diagnosis in whole slide images of core needle biopsies: a blinded clinical validation and deployment study. The Lancet Digital Health 2(8): e407-16

Patcas R, Timofte R, Volokitin A, Agustsson E, Eliades T, Eichenberger M, Bornstein MM (2019) Facial attractiveness of cleft patients: a direct comparison between artificial-intelligence-based scoring and conventional rater groups. Eur J Orthod 41(4):428-433

Patel D, Kore SA (2020) Artificial intelligence: future impacts, challenges and recommendations on healthcare services. Int J Community Med Public Heal 7(4):1596

Pathak, Anil D., et al. (2021) Smart war on COVID-19 and global pandemics: integrated $\mathrm{AI}$ and blockchain ecosystem.Computational modeling and data analysis in COVID-19 research. CRC Press: 67-94.

Paul D, Sanap G, Shenoy S, Kalyane D, Kalia K, Tekade RK (2021) Artificial intelligence in drug discovery and development. Drug Discov Today 26:80-93

Peter Behner et al. (2017) Fighting counterfeit pharmaceuticals: new defenses for an underestimated - and growing - menace", Strategy.

Peng QY et al.(2020) Findings of lung ultrasonography of novel corona virus pneumonia during the 2019 - 2020 epidemic. Intensive Care Med: 6-7.

Pereira JC (2016) Boosting docking-based virtual screening with deep learning. J Chem Inf Model 56:2495-2506

Pianykh OS, Langs G, Dewey M et al (2020) Continuous learning AI in radiology: implementation principles and early applications. Radiology 297(1):6-14

Pires DEV, Blundell TL, Ascher DB (2015) pkCSM: Predicting smallmolecule pharmacokinetic and toxicity properties using graph-based signatures. J Med Chem 58:4066-4072

Prosser M (2020) How AI helped predict the coronavirus coutbreak before it happened. Singularity Hub.

Rallapalli S (2016) Improving healthcare-big data analytics for electronic health records on cloud. J Adv Inf Technol 7(1):65-68 
Rao ASS, Vazquez JA (2020) Identification of COVID-19 can be quicker through artificial intelligence framework using a mobile phonebased survey when cities and towns are under quarantine. Infect Control Hosp Epidemiol 41(7):826-830

Rosebrock A (2020) Detecting COVID-19 in X-ray images with Keras. TensorFlow, and Deep Learning

Sacleux, S. C., \& Samuel, D. (2019) A critical review of MELD as a reliable tool for transplant prioritization. In Seminars in liver disease (Vol. 39, No. 04, pp. 403-413). Thieme Medical Publishers.

Sahoo MS, Baruah PK (2018) HBasechainDB - a scalable blockchain framework on Hadoop Ecosystem. In: Yokota R., Wu W. (eds) Supercomputing frontiers. SCFA 2018. Lecture notes in computer science, vol 10776. Springer, Cham.

Scekic O, Nastic S, Dustdar S (2019) Blockchain-supported smart city platform for social value co-creation and exchange. IEEE Internet Comput 23(1):19-28

Schechtman D (2019) Introdução e Guia Prático a Smart Contracts (Introduction and practical guide to smart contracts). SSRN Electron J 3317504

Singh M, Kim S (2018) Branch based blockchain technology in intelligent vehicle. Comput Netw 145:219-231

Siyal AA, Junejo AZ, Zawish M, Ahmed K, Khalil A, Soursou G (2019) Applications of blockchain technology in medicine and healthcare: challenges and future perspectives. Cryptography 3(1):3

Taylor D (2016) The pharmaceutical industry and the future of drug development. Pharmaceuticals in the environment. In: Environ Sci Technol Wiley 2015:1-33

Tran V-T, Riveros C, Ravaud P (2019) Patients' views of wearable devices and $\mathrm{AI}$ in healthcare: findings from the ComPaRe e-cohort. NPJ Digit Med 2(1):1-8

Timón S, Rincón M, Martínez-Tomás R (2017) Extending xnat platform with an incremental semantic framework. Front Neuroinform 11:57

Uchiyama S, Hoshino T, Sissani L, Linsay MT, Kamiyama K, Nakase T, Kitagawa K, Minematsu K, Todo K, Okada Y, Nakagawara J, Nagata K, Yamagami H, Yamaguchi T, Amarenco P, TIAregistry.org Investigators (2019) Japanese versus non-Japanese patients with transient ischemic attack or minor stroke: subanalysis of TIA registry. org. J Stroke Cerebrovasc Dis 28(8):2232-2241

Van der V, Bas HM et al (2017) Complementary value of contralateral parenchymal enhancement on DCE-MRI to prognostic models and molecular assays in high-risk ER+/HER2- breast cancer. Clin Cancer Res 23(21):6505-6515

Vopham T, Hart JE, Laden F, Chiang YY (2018) Emerging trends in geospatial artificial intelligence (geoAI): Potential applications for environmental epidemiology. Environ Heal A Glob Access Sci Source 17(1):1-6

Wang S, Wang J, Wang X, Qiu T, Yuan Y, Ouyang L, Guo Y, Wang FY (2018) Blockchain-powered parallel healthcare systems based on the ACP approach. IEEE Trans Comput Soc Syst 5(4):942-950

Wang H, Song Y (2018) Secure cloud-based EHR system using attributebased cryptosystem and blockchain. J Med Syst 42(8):1-9
Wang C, Zhang Y (2017) Improving scoring-docking-screening powers of protein-ligand scoring functions using random forest. J Comput Chem 38(3):169-177

Wahl B, Cossy-Gantner A, Germann S, Schwalbe NR (2018) Artificial intelligence (AI) and global health: how can AI contribute to health in resource-poor settings? BMJ Glob Health 3(4):e000798

Wong ZSY, Zhou J, Zhang Q (2019) Artificial intelligence for infectious disease. Big data analytics. Infect Dis Heal 24(1):44-48

Xia Q, Sifah EB, Asamoah KO, Gao J, du X, Guizani M (2017) MeDShare: trust-less medical data sharing among cloud service providers via blockchain. IEEE Access 5:14757-14767

Xing L, Kapp DS, Giger ML, Min JK (2021) Outlook of the future landscape of artificial intelligence in medicine and new challenges. In: Artificial intelligence in medicine: 503-526.

Yip TC-F, Chan HL-Y, Tse Y-K, Lam KL-Y, Lui GC-Y, Wong VW-S, Wong GL-H (2018) On-treatment improvement of MELD score reduces death and hepatic events in patients with hepatitis Brelated cirrhosis. Off J Am College Gastroenterol| ACG 113(11): $1629-1638$

Yue X, Wang H, Jin D, Li M, Jiang W (2016) Healthcare Data Gateways: found healthcare intelligence on blockchain with novel privacy risk control. J Med Syst 40(10):218

Zhang GZ, Deng YJ, Xie QQ, Ren EH, Ma ZJ, He XG, Gao YC, Kang XW (2020) Sirtuins and intervertebral disc degeneration: roles in inflammation, oxidative stress, and mitochondrial function. Clin Chim Acta 508:33-42

Zhang P, White J, Schmidt DC, Lenz G, Rosenbloom ST (2018a) FHIRChain: applying blockchain to securely and scalably share clinical data. Comput Struct Biotechnol J 16:267-278

Zhou L, Wang L, Sun Y (2018) MIStore: a blockchain-based medical insurance storage system. J Med Syst: 42

Zhang P, White J, Schmidt DC, Lenz G, Rosenbloom ST (2018b) FHIRChain: applying blockchain to securely and scalably share clinical data. Comput Struct Biotechnol J 16:267-278

Zhu H (2020) Big data and artificial intelligence modeling for drug discovery. Annu Rev Pharmacol Toxicol 60:573-589

Zhang L (2017) From machine learning to deep learning: progress in machine intelligence for rational drug discovery. Drug Discov Today 22:1680-1685

Zou FW, Tang YF, Liu CY, Ma JA, Hu CH (2020) Concordance study between IBM Watson for oncology and real clinical practice for cervical cancer patients in China: a retrospective analysis. Front Genet 11

Zoete V, Daina A, Bovigny C, Michielin O (2016) SwissSimilarity: a web tool for low to ultra high throughput ligand-based virtual screening. J Chem Inf Model 56:1399-1404

Publisher's note Springer Nature remains neutral with regard to jurisdictional claims in published maps and institutional affiliations. 